\title{
Charge-and-Energy Conserving Moment-Based Accelerator for a Multi-Species Vlasov-Fokker-Planck-Ampère System, Part I: Collisionless Aspects
}

\author{
William T. Taitano, Luis Chacón \\ Los Alamos National Laboratory, Los Alamos, NM, USA
}

\begin{abstract}
In this study, we propose a charge, momentum, and energy conserving discretization for the 1D-1V VlasovAmpère system of equations on an Eulerian grid. The new conservative discretization is nonlinear in nature, but can be efficiently converged with a moment-based nonlinear accelerator algorithm. We demonstrate the conservation and convergence properties of the scheme with various numerical examples, including a multi scale ion-acoustic shockwave problem.
\end{abstract}

\section{Introduction}

The dynamic evolution of coupled electromagnetic fields and collisionless plasmas is described by the VlasovMaxwell system. The Maxwell equations evolve the self-consistent fields based on moments of the ion-electron distribution functions (charge and current densities). The plasma distribution function evolution is governed by the Vlasov equation, which is coupled back to the Maxwell system through electromagnetic fields. In this study, we will focus on a deterministic solution approach for this nonlinearly coupled system.

Historically, explicit and operator-split-based methods have been used to model collisionless kinetic plasmas $[1,2,3,5,7]$. The explicit method is massively parallelizable, simple to implement and is computationally inexpensive per time step. However, the method is hampered by the well-known CFL stability limit for a given cell size of the problem. For a multi length-scale and time-scale problem, this limitation makes the explicit method inefficient. On the other hand, operator-split-based semi-implicit methods are not limited by the CFL limitations and allow larger time-steps $[\mathbf{5}, \mathbf{3}, \mathbf{4}]$. However, for long time-scale integrations, low-order splitting errors can accumulate and become significant. Additionally, most approaches to date lack higher-order discrete conservation properties such as momentum and energy, and can accumulate large conservation errors for long-time integrations $[\mathbf{2 4}, \mathbf{2 5}]$.

In contrast, a fully implicit, unsplit approach for the Vlasov-Ampère/Poisson system aims to converge the nonlinear residual to a tight tolerance within a time-step. By converging the nonlinear system to a tight tolerance, much larger time-steps can be used without accumulating large errors from time-step to time-step particularly when exact conservation properties are enforced. In this study, we put forth a modern, fully implicit method which accelerates the convergence of the nonlinearly coupled Vlasov-Ampère system. The algorithm is based on a new, discretely consistent implicit moment method (IMM) [15] for the Eulerian approach. The new IMM scheme is based on a high-order (HO) low-order (LO) nonlinear convergence acceleration method which has seen success in neutron transport [13], thermal radiation transport [14], neutral gas kinetics [12], and collisionless plasma physics (using a PIC approach [15]). In the proposed algorithm, a LO moment-field system is used to accelerate the convergence of the HO Vlasov-Ampère system. The LO system provides the HO system with the accelerated evaluation of the electric field for the Vlasov operator. In turn, the HO system provides the LO system with the closure at the total stress tensor level.

Sophisticated approaches in both implicit and explicit schemes have been developed to enforce exact conservation properties. In the context of particle-in-cell (PIC) approaches, a fully implicit algorithm for the Vlasov-Ampère system has been demonstrated to conserve charge and energy exactly in the discrete [23]. A recent study [8] proposes an implicit energy conserving discretization for an Eulerian-based Vlasov-Ampère 
system. Additionally, a novel explicit method has been developed which conserves both momentum and energy in the limit of $\Delta t \rightarrow 0$ using a variational formulation of the Vlasov-Ampère system and a truncated Fourier basis [9]. However, to our knowledge, a finite volume/difference scheme for the Vlasov-Ampère system which conserves charge, momentum, and energy for finite $\Delta t$ does not yet exist. In this study, we develop a novel finite volume-based nonlinear discretization scheme which achieves simultaneous conservation of charge, momentum, and energy by enforcing two additional set of discrete nonlinear constraints.

The remainder of the manuscript is organized as follows. Section 2 introduces the governing set of equations for a one spatial dimension and one velocity space dimension (1D1V) Vlasov-Ampère system. The derivation of the moment system is presented and a general flavor of the HOLO algorithm is provided. In section 3, we discuss the charge, momentum, and energy conserving discretization of the coupled VlasovAmpère system and introduce the required set of discrete nonlinear constraints. Section 4 discusses the solver method used for both the HO kinetic and LO moment-field systems. Finally, in section 5, we demonstrate the discrete conservation properties and the performance of the HOLO moment-based acceleration algorithm via numerical experiment. Concluding remarks are provided in section 6.

\section{HOLO System of Equations for the Vlasov-Ampère System}

The moment acceleration technique we propose in this study is a direct extension of the method developed for the particle-in-cell (PIC) approach in reference [15]. The method is based on a coupled, higher dimensional (HO) kinetic system and lower dimensional (LO) moment-field system. The solution of the LO system takes the HO stress tensor to solve for the implicit electric field based on the moment equations. The HO system employs the new electric field from the LO system and returns the self-consistent stress-tensor closure to the LO system.

For a proof of principle study, a 1D configuration space and 1D velocity space (1D1V), electrostatic, two-species Vlasov-Ampère formulation is considered:

$$
\begin{gathered}
\frac{\partial f_{e}}{\partial t}+v \frac{\partial f_{e}}{\partial x}+\frac{q_{e}}{m_{e}} E \frac{\partial f_{e}}{\partial v}=0 \\
\frac{\partial f_{i}}{\partial t}+v \frac{\partial f_{i}}{\partial x}+\frac{q_{i}}{m_{i}} E \frac{\partial f_{i}}{\partial v}=0 \\
\epsilon_{0} \frac{\partial E}{\partial t}+\sum_{\alpha}^{N_{s}} q_{\alpha} n u_{\alpha}=0 .
\end{gathered}
$$

Equations (2.1), (2.2), and (2.3) are the electron and ion Vlasov equations and the Ampère's equation, respectively. In these equations, $m_{e}, m_{i}, f_{e}, f_{i}, v, q_{e}, q_{i}, E, \epsilon_{0}$, and $n u_{\alpha}$ are the electron and ion mass, electron and ion distribution function, electron and ion velocity, electron and ion charge, the electric field, permittivity constant of vacuum, and the number density flux of species $\alpha$, respectively. The Vlasov equation is a function of position, velocity, and time. The Ampère's equation is solved for the self-consistent electric field, which is a function of space and time. The coupled set of nonlinear equations are solved fully implicitly. Again for this study, a deterministic, Eulerian approach will be adopted to discretize the Vlasov equation.

\subsection{Derivation of the coupled moment/Vlasov-Ampère System}

In this section, we formulate the HO Vlasov equation, and the LO moment-field equations which are used to accelerate the nonlinear convergence of the Vlasov-Ampére system. The Vlasov equation for species $\alpha$, $\left(\mathrm{VE}_{\alpha}\right)$, is defined according to equations (2.1) and (2.2) as:

$$
\mathrm{VE}_{\alpha}=\frac{\partial f_{\alpha}}{\partial t}+v \frac{\partial f_{\alpha}}{\partial x}+\frac{q_{\alpha}}{m_{\alpha}} E \frac{\partial f_{\alpha}}{\partial v}=0
$$

We define the $l^{\text {th }}$ moment of the velocity distribution function of species $\alpha$ as follows,

$$
\mathcal{M}_{\alpha}^{l}=\int_{-\infty}^{+\infty} v^{l} f_{\alpha} d v=\left\langle v^{l}, f_{\alpha}\right\rangle_{v}
$$


With this definition, the number density, $n_{\alpha}$, number density flux, $n u_{\alpha}$, and total stress-tensor, $S_{\alpha}$ are the $0^{t h}, 1^{\text {st }}$, and $2^{\text {nd }}$ moments of the distribution function, respectively, and can be written as:

$$
\begin{gathered}
n_{\alpha}=\left\langle 1, f_{\alpha}\right\rangle_{v}, \\
n u_{\alpha}=\left\langle v, f_{\alpha}\right\rangle_{v}, \\
S_{\alpha}=\left\langle v^{2}, f_{\alpha}\right\rangle_{v} .
\end{gathered}
$$

The governing equations for the fluid moments (LO problem) are obtained by taking the $0^{\text {th }}$, and $1^{\text {st }}$ moment of the Vlasov equation to find:

$$
\begin{gathered}
\langle 1, \mathrm{VE}\rangle_{v}=\frac{\partial n_{\alpha}}{\partial t}+\frac{\partial}{\partial x} n u_{\alpha}=0 \\
\langle v, \mathrm{VE}\rangle_{v}=\frac{\partial}{\partial t} n u_{\alpha}+\frac{\partial}{\partial x} S_{\alpha}-\frac{q_{\alpha}}{m_{\alpha}} n_{\alpha} E=0 .
\end{gathered}
$$

Equations (2.9) and (2.10) are the continuity and momentum conservation equations, respectively, for each species. We consider the coupled HO kinetic system and a LO moment-field system for an ion-electron system:

HO (Kinetic) System:

$$
\begin{gathered}
\frac{\partial f_{e}}{\partial t}+v \frac{\partial f_{e}}{\partial x}+\frac{q_{e}}{m_{e}} E \frac{\partial f_{e}}{\partial v}=0 \\
\frac{\partial f_{i}}{\partial t}+v \frac{\partial f_{i}}{\partial x}+\frac{q_{i}}{m_{i}} E \frac{\partial f_{i}}{\partial v}=0
\end{gathered}
$$

LO (Moment-Field) System:

$$
\begin{gathered}
\frac{\partial n_{e}}{\partial t}+\frac{\partial}{\partial x} n u_{e}^{L O}=0 \\
\frac{\partial}{\partial t} n u_{e}^{L O}+\frac{\partial}{\partial x} n_{e}^{L O} \widetilde{S}_{e}^{H O}-\frac{q_{e}}{m_{e}} n_{e}^{L O} E=\gamma_{n u_{e}}^{H O} \\
\frac{\partial n_{i}{ }^{L O}}{\partial t}+\frac{\partial}{\partial x} n u_{i}^{L O}=0 \\
\frac{\partial}{\partial t} n u_{i}^{L O}+\frac{\partial}{\partial x} n_{i}^{L O} \widetilde{S}_{i}^{H O}-\frac{q_{i}}{m_{i}} n_{i}^{L O} E=\gamma_{n u_{i}}^{H O} \\
\epsilon_{0} \frac{\partial E}{\partial t}+\sum_{\alpha}^{N_{s}} q_{\alpha} n u_{\alpha}^{L O}=0 .
\end{gathered}
$$

The closure required for the LO fluid moment system is provided by the HO density-normalized total stresstensor $\widetilde{S}_{\alpha}^{H O}$,

$$
\widetilde{S}_{\alpha}^{H O}=\frac{\left\langle v^{2}, f_{\alpha}\right\rangle_{v}}{\left\langle 1, f_{\alpha}\right\rangle_{v}},
$$

and the discrete consistency term, $\gamma_{\alpha}^{H O}[15]$. The purpose of the density normalization is to expose the stiff isothermal wave in the LO system [15]. In doing so, we enhance the convergence rate of the HO-LO iteration within a time step when the time step is large compared to the stiff acoustic time-scales. We introduce the discrete consistency terms and their purpose in the next section. 


\subsection{Discrete Consistency Terms}

As discussed in the preceding section, the $\mathrm{HO}$ and LO systems are exactly equivalent in the continuum when the exact closures for $S_{\alpha}^{H O}$ and $E$ are employed. However, an issue arises in the discrete. Upon discretization of the HO and LO system of equations, discretization errors arise in both systems. While we cannot avoid discretization error, we also cannot tolerate the discretization error of the two systems (HO and LO) evolving separately. If such situation arises, then, over many time steps, the two systems will drift apart, leading to inaccurate solution and solver breakdown. The purpose of $\gamma_{n u_{\alpha}}^{H O}$ is to enslave the discretization error of the LO system to the discretization error of the HO system [15]. The continuum expression for $\gamma_{n u_{\alpha}}^{H O}$ is given as,

$$
\gamma_{n u_{\alpha}}^{H O}=\frac{\partial}{\partial t} n u_{\alpha}^{H O}+\frac{\partial}{\partial x} n_{\alpha}^{H O} \widetilde{S}_{\alpha}^{H O}-\frac{q_{\alpha}}{m_{\alpha}} n_{\alpha}^{H O} E
$$

The HO moment variables are calculated by numerical integration of the HO solution to $f_{\alpha}$. In this study, the moments are calculated as:

$$
\begin{gathered}
n_{\alpha}^{H O}=\left\langle 1, f_{\alpha}\right\rangle_{v} \approx \sum_{j}^{N_{v}} \Delta v_{\alpha_{j}} f_{\alpha_{j}}, \\
n u_{\alpha}^{H O}=\left\langle v, f_{\alpha}\right\rangle_{v} \approx \sum_{j}^{N_{v}} \Delta v_{\alpha_{j}} v_{\alpha_{j}} f_{\alpha_{j}}, \\
\widetilde{S}_{\alpha}^{H O}=\frac{\left\langle v^{2}, f_{\alpha}\right\rangle_{v}}{\left\langle 1, f_{\alpha}\right\rangle_{v}} \approx \frac{\sum_{j}^{N_{v}} \Delta v_{\alpha_{j}} v_{\alpha_{j}}^{2} f_{\alpha_{j}}}{\sum_{j}^{N_{v}} \Delta v_{\alpha_{j}} f_{\alpha_{j}}} .
\end{gathered}
$$

Here, $j$ is the discrete velocity space grid point, $\Delta v_{\alpha_{j}}$ is the $j^{t h}$ discrete velocity space size for species $\alpha, N_{v}$ is the number of velocity space cell, $v_{\alpha_{j}}$ is the $j^{\text {th }}$ discrete velocity space location, and $f_{\alpha_{j}}$ is the distribution function at the $j^{t h}$ discrete velocity space grid point. When $\gamma_{n u_{\alpha}}^{H O}$ is evaluated in this manner, and then used in (2.14) and (2.16), discrete consistency is enforced upon convergence of the HO-LO iteration. We only require the consistency term, $\gamma_{n u_{\alpha}}^{H O}$ in the $1^{s t}$ moment equation and not in the $0^{t h}$ moment equation. This is so because we choose a conservative, finite-volume discretization of the Vlasov equation in this study, which will trivially enforce the discrete $0^{t h}$ moment equation (i.e. the consistency term automatically evaluates to zero).

\section{Charge, Momentum, and Energy Conserving Discretization}

In what follows, we consider a periodic system in physical space. We begin by fully discretizing the VlasovAmpère system on a staggered mesh,

$$
\begin{gathered}
\mathrm{VE}_{\alpha_{i, j}}^{k+1}=\frac{f_{\alpha_{i, j}}^{k+1}-f_{\alpha_{i, j}}^{k}}{\Delta t}+v_{\alpha_{j}} \frac{\widehat{f}_{\alpha_{i+1 / 2, j}}^{k+1 / 2}-\widehat{f}_{\alpha_{i-1 / 2, j}}^{k+1 / 2}}{\Delta x_{i}}+\frac{q_{\alpha}}{m_{\alpha}} E_{i}^{k+1 / 2} \frac{\widetilde{f}_{\alpha_{i, j}+1 / 2}^{k+1 / 2}-\widetilde{f}_{\alpha_{i, j-1 / 2}}^{k+1 / 2}}{\Delta v_{\alpha_{j}}}=0, \\
\epsilon_{0} \frac{E_{i+1 / 2}^{k+1}-E_{i+1 / 2}^{k}}{\Delta t}+\sum_{\alpha}^{N_{s}} q_{\alpha} n u_{\alpha_{i+1 / 2}}^{k+1 / 2}=0,
\end{gathered}
$$

where we have used a Crank-Nicolson discretization in time and a finite-volume discretization in space and velocity. Here $i$ is the spatial cell index, $j$ is the velocity space cell index, $k$ is the time index, $\Delta t$ is the time-step-size, $\Delta x_{i}$ is the cell size of the $i^{t h}$ spatial cell, $\Delta v_{\alpha_{j}}$ is the velocity space cell size of the $j^{t h}$ velocity space cell for the $\alpha$ species, $E_{i+1 / 2}^{k+1}$ is the cell-face electric field, $E_{i}^{k+1 / 2}$ is the time-centered cell-center electric field,

$$
E_{i}^{k+1 / 2}=\frac{E_{i+1 / 2}^{k+1 / 2}+E_{i-1 / 2}^{k+1 / 2}}{2}=\frac{\left(E_{i+1 / 2}^{k+1}+E_{i+1 / 2}^{k}\right)+\left(E_{i-1 / 2}^{k+1}+E_{i-1 / 2}^{k}\right)}{4},
$$




\begin{tabular}{|c|c|c|c|}
\hline & Charge Cons. & Momentum Cons. & Energy Cons. \\
\hline$n_{\alpha_{i}}^{k+1 / 2}=\sum_{j}^{N v} \Delta v_{\alpha_{j}} f_{\alpha_{i, j}}^{k+1 / 2}$ & Yes & No & N/A \\
\hline$n_{\alpha_{i}}^{k+1 / 2}=\sum_{j}^{N v} \Delta v_{\alpha_{j+1 / 2}} \widetilde{f}_{\alpha_{i, j}+1 / 2}^{k+1 / 2}$ & No & Yes & N/A \\
\hline$n u_{\alpha_{i+1 / 2}}^{k+1 / 2}=\sum_{j}^{N v} \Delta v_{\alpha_{j}} v_{\alpha_{j}} \widehat{f}_{\alpha_{i+1 / 2, j}}^{k+1 / 2}$ & Yes & Yes & No \\
\hline$n u_{\alpha_{i+1 / 2}}^{k+1 / 2}=\frac{\sum_{j}^{N v} \Delta v_{\alpha_{j+1 / 2}} v_{\alpha_{j+1 / 2}}\left(\Delta x_{i} \tilde{f}_{\left.\alpha_{i, j+1 / 2}+\Delta x_{i+1} \tilde{f}_{\alpha_{i+1, j}+1 / 2}^{k+1 / 2}\right)}^{2 \Delta x_{i+1 / 2}}\right.}{2}$ & No & No & Yes \\
\hline
\end{tabular}

Table 1: Choice of moments and their associated conservation properties.

$\widehat{f}_{\alpha_{i+1 / 2, j}}^{k+1 / 2}$ is the time-centered cell-face configuration space numerical flux for species $\alpha$,

$$
\widehat{f}_{\alpha_{i+1 / 2, j}}^{k+1 / 2}=\frac{1}{2}\left(\widehat{f}_{\alpha_{i+1 / 2, j}}^{k+1}+\widehat{f}_{\alpha_{i+1 / 2, j}}^{k}\right),
$$

and $\widetilde{f}_{\alpha_{i, j+1 / 2}}^{k+1 / 2}$ is the time-centered cell-face velocity space numerical flux for species $\alpha$,

$$
\widetilde{f}_{\alpha_{i, j+1 / 2}}^{k+1 / 2}=\frac{1}{2}\left(\widetilde{f}_{\alpha_{i, j+1 / 2}}^{k+1}+\widetilde{f}_{\alpha_{i, j+1 / 2}}^{k}\right) .
$$

We do not wish to specify the definition of $n u_{\alpha_{i+1 / 2}}^{k+1 / 2}$ in terms of $f_{\alpha_{i, j}}^{k+1}$ and $f_{\alpha_{i, j}}^{k}$ yet.

In order to enforce separately the conservation of charge, momentum, and energy, particular definitions of density, $n$, and number density flux, $n u$, are required. These definitions are derived in Appendix B, and are organized in Table 1. As can be seen, the definitions of $n$ and $n u$ required for the separate conservation properties are mutually inconsistent. In order to enforce simultaneous conservation of charge, momentum, and energy, one must enforce a set of discrete nonlinear constraints that arise from analyzing the individual discrete conservation properties. The proposed discretization scheme which enforce simultaneous charge, momentum, and energy conservation requires the following modification to the Vlasov-Ampère system:

$$
\begin{aligned}
& \frac{f_{\alpha_{i, j}}^{k+1}-f_{\alpha_{i, j}}^{k}}{\Delta t}+\underbrace{v_{\alpha_{j}} \frac{\widehat{f}_{\alpha_{i+1 / 2, j}}^{k+1 / 2}-\widehat{f}_{\alpha_{i-1 / 2 j}}^{k+1}}{\Delta x_{i}}}_{\text {(a) }}+\underbrace{\left|v_{\alpha_{j}}\right| \frac{\xi_{\alpha_{i+1 / 2}}^{k+1} \hat{f}_{\alpha_{i+1 / 2, j}}^{k+1, *}-\xi_{\alpha_{i-1 / 2}}^{k+1} \widehat{f}_{\alpha_{i-1 / 2, j}}^{k+1, *}}{\Delta x_{i}}}_{\text {(D) }}+ \\
& \frac{q_{\alpha}}{m_{\alpha}} E_{i}^{k+1 / 2}(\underbrace{\frac{\widetilde{f}_{\alpha_{i, j}}^{k+1 / 2}-\widetilde{f}_{\alpha_{i, j-1 / 2}}^{k+1 / 2}}{\Delta v_{\alpha_{j}}}}_{\text {(C) }}+\underbrace{\phi_{\alpha_{i}}^{k+1} \frac{\widetilde{f}_{\alpha_{i, j}+1 / 2}^{k+1, *}-\widetilde{f}_{\alpha_{i, j-1 / 2}}^{k+1, *}}{\Delta v_{\alpha_{j}}}}_{\text {(d) }})=0, \\
& \epsilon_{0} \frac{E_{i+1 / 2}^{k+1}-E_{i+1 / 2}^{k}}{\Delta t}+\sum_{\alpha}^{N_{s}} q_{\alpha} n u_{E C, \alpha_{i+1 / 2}}^{k+1 / 2}=0 .
\end{aligned}
$$

Here, terms (a) and (C) are the discrete physical streaming and acceleration operators, respectively, and (b) and (d) are referred to as the charge conserving, and momentum conserving consistency operators, respectively. $\xi_{\alpha_{i+1 / 2}}^{k+1}$ and $\phi_{\alpha_{i}}^{k+1}$ are nonlinear coefficients which enforces the set of nonlinear constraints, and are defined as:

$$
\xi_{\alpha_{i+1 / 2}}^{k+1}=\frac{n u_{E C, \alpha_{i+1 / 2}}^{k+1 / 2}-\widehat{n u}_{\alpha_{i+1 / 2}}^{k+1 / 2}}{\sum_{j}^{N_{v}} \Delta v_{\alpha_{j}}\left|v_{\alpha_{j}}\right| \widehat{f}_{\alpha_{i+1 / 2, j}}^{k+1, *}} .
$$

and

$$
\phi_{\alpha_{i}}^{k+1}=\frac{n_{\alpha_{i}}^{k+1 / 2}-\widetilde{n}_{\alpha_{i}}^{k+1 / 2}}{\sum_{j}^{N_{v}} \Delta v_{j+1 / 2} \widetilde{f}_{\alpha_{i, j+1 / 2}}^{k+1, *}},
$$


where,

$$
\begin{gathered}
\widehat{n u}_{\alpha_{i+1 / 2}}^{k+1 / 2}=\sum_{j}^{N_{v}} \Delta v_{\alpha_{j}} v_{\alpha_{j}} \widehat{f}_{\alpha_{i+1 / 2, j}}^{k+1 / 2}, \\
n u_{E C, \alpha_{i+1 / 2}}^{k+1 / 2}=\frac{\sum_{j}^{N_{v}} \Delta v_{\alpha_{j+1 / 2}} v_{\alpha_{j+1 / 2}}\left[\Delta x_{i}\left(\widetilde{f}_{\alpha_{i, j+1 / 2}}^{k+1 / 2}+\phi_{\alpha_{i}}^{k+1} \widetilde{f}_{\alpha_{i, j+1 / 2}}^{k+1, *}\right)+\Delta x_{i+1}\left(\widetilde{f}_{\alpha_{i+1, j+1 / 2}}^{k+1 / 2}+\phi_{\alpha_{i+1}}^{k+1} \widetilde{f}_{\alpha_{i, j+1 / 2}}^{k+1, *}\right)\right]}{2 \Delta x_{i+1 / 2}}, \\
n_{\alpha_{i}}^{k+1 / 2}=\sum_{j}^{N_{v}} \Delta v_{\alpha_{j}} f_{\alpha_{i, j}}^{k+1 / 2},
\end{gathered}
$$

and

$$
\widetilde{n}_{\alpha_{i}}^{k+1 / 2}=\sum_{j}^{N_{v}} \Delta v_{\alpha_{j+1 / 2}} \widetilde{f}_{\alpha_{i, j+1 / 2}}^{k+1 / 2} .
$$

Additionally, $\widehat{f}_{\alpha_{i+1 / 2, j}}^{k+1, *}$ is the numerical flux at the configuration space cell face for the charge conserving consistency operator, and $\widetilde{f}_{\alpha_{i, j+1 / 2}}^{k+1, *}$ is the numerical flux at velocity space cell face for the momentum conserving consistency operator. The definitions and purpose of the additional terms are discussed in the next sections.

\subsection{Charge-and-Momentum Conserving Discretization}

From Table 1 and the analysis of individual discrete conservation properties (Appendix B), it is clear that the definitions of $n_{\alpha_{i}}^{k+1 / 2}$ required for charge conservation and momentum conservation are not guaranteed to be consistent. Thus, in general, one will not achieve simultaneous conservation of charge and momentum. To enforce conservation, we introduce an additional nonlinear constraint to deal with this inconsistency. First, we present the modification of the Vlasov equation which enforces both charge and momentum conservation:

$$
\begin{array}{r}
\frac{f_{\alpha_{i, j}}^{k+1}-f_{\alpha_{i, j}}^{k}}{\Delta t}+v_{\alpha_{j}} \frac{\widehat{f}_{\alpha_{i+1 / 2, j}}^{k+1 / 2}-\widehat{f}_{\alpha_{i-1 / 2, j}}^{k+1 / 2}}{\Delta x_{i}} \\
\frac{q_{\alpha}}{m_{\alpha}} E_{i}^{k+1 / 2}(\widetilde{f}_{{\tilde{\alpha_{i, j}}}^{k+1 / 2}-\widetilde{f}_{\alpha_{i, j-1 / 2}}^{k+1 / 2}}^{\Delta+1 / 2}+\underbrace{\phi_{\alpha_{i}}^{k+1} \frac{\widetilde{f}_{\alpha_{i, j}}^{k+1, *}-\widetilde{f}_{\alpha_{i, j-1 / 2}}^{k+1, *}}{\Delta v_{\alpha_{j}}}}_{\text {(a) }})=0 .
\end{array}
$$

Here, term (a) is a truncation error term which enforces that the momentum-conserving number density be equal to the charge-conserving number density. In this term, the coefficient $\phi_{\alpha_{i}}^{k+1}$ will enforce the nonlinear constraint, and $\widetilde{f}_{\alpha_{i, j+1 / 2}}^{k+1, *}$ is the numerical flux at the cell face. The $*$ simply denotes that the choice of numerical flux discretization can be different from that of the physical acceleration operator. The purpose of (a) is to correct the truncation error in the conservation properties introduced by the discretization of the physical operator. Hence, the consistency term (a) will naturally be of the order truncation error of the physical operator, regardless of the discretization used for $\widehat{f}^{*}$. It follows that the (a) term will not change the order of accuracy of the discretization. This will be shown numericallylater in this study.

In order to determine the expression for $\phi_{\alpha_{i}}^{k+1}$, we introduce the following nonlinear constraint,

$$
\underbrace{\widetilde{n}_{\alpha_{i}}^{k+1 / 2}+\Phi_{\alpha_{i}}^{k+1}}_{\text {momentum conserving density }}=\underbrace{n_{\alpha_{i}}^{k+1 / 2}}_{\text {charge conserving density }},
$$


where,

$$
\begin{gathered}
n_{\alpha_{i}}^{k+1 / 2}=\frac{\sum_{j}^{N_{v}} \Delta v_{j}\left(f_{\alpha_{i, j}}^{k+1}+f_{\alpha_{i, j}}^{k}\right)}{2}, \\
\widetilde{n}_{\alpha_{i}}^{k+1 / 2}=\sum_{j}^{N_{v}} \Delta v_{j+1 / 2} \widetilde{f}_{\alpha_{i, j}+1 / 2}^{k+1 / 2},
\end{gathered}
$$

and

$$
\Phi_{\alpha_{i}}^{k+1}=\phi_{\alpha_{i}}^{k+1} \sum_{j}^{N_{v}} \Delta v_{j+1 / 2} \widetilde{f}_{\alpha_{i, j+1 / 2}}^{k+1, *} .
$$

From equations (3.10), (3.11), and (3.12), we can solve for $\phi_{\alpha_{i}}^{k+1}$ as,

$$
\phi_{\alpha_{i}}^{k+1}=\frac{n_{\alpha_{i}}^{k+1 / 2}-\widetilde{n}_{\alpha_{i}}^{k+1 / 2}}{\sum_{j}^{N_{v}} \Delta v_{j+1 / 2} \widetilde{f}_{\alpha_{i, j+1 / 2}}^{k+1, *}} .
$$

Hence the discretization is inherently nonlinear, and requires an iterative scheme to converge the resulting system. However, this is not a disadvantage in our algorithm, as the nonlinearity in the discretization can be absorbed in the HOLO iteration. We note that $\Phi_{\alpha_{i}}^{k+1}$ is a measure of discretization truncation error difference between $n_{\alpha_{i}}^{k+1 / 2}$ and $\widetilde{n}_{\alpha_{i}}^{k+1 / 2}$, and therefore $\Phi_{\alpha_{i}}^{k+1}=0$ in the continuum.

\subsection{Charge-and-Energy Conserving Discretization}

As in the previous section, the discrete definitions of $n u_{\alpha_{i+1 / 2}}^{k+1 / 2}$ required for charge and energy conservation are inconsistent. Again, we introduce an additional nonlinear constraint to deal with this discrete inconsistency. First, we present the modification of the Vlasov-Ampère system which will enforce both charge and energy conservation,

$$
\begin{aligned}
& \frac{f_{\alpha_{i, j}}^{k+1}-f_{\alpha_{i, j}}^{k}}{\Delta t}+v_{\alpha_{j}} \frac{\widehat{f}_{\alpha_{i+1 / 2, j}}^{k+1 / 2}-\widehat{f}_{\alpha_{i-1 / 2, j}}^{k+1 / 2}}{\Delta x_{i}}+\underbrace{\left|v_{\alpha_{j}}\right| \frac{\xi_{\alpha_{i+1 / 2}}^{k+1} \widehat{f}_{\alpha_{i+1 / 2, j}}^{k+1, *}-\xi_{\alpha_{i-1 / 2}}^{k+1} \widehat{f}_{\alpha_{i-1 / 2, j}}^{k+1, *}}{\Delta x_{i}}}_{\text {(a) }}+ \\
& \frac{q_{\alpha}}{m_{\alpha}} E_{i}^{k+1 / 2} \frac{\widetilde{f}_{\alpha_{i, j+1 / 2}}^{k+1 / 2}-\widetilde{f}_{\alpha_{i, j-1 / 2}}^{k+1 / 2}}{\Delta v_{\alpha_{j}}}=0, \\
& \epsilon_{0} \frac{E_{i+1 / 2}^{k+1}-E_{i+1 / 2}^{k}}{\Delta t}+\sum_{\alpha}^{N_{s}} q_{\alpha} \widetilde{n u} \alpha_{\alpha_{i+1 / 2}}^{k+1 / 2}=0 .
\end{aligned}
$$

Here, (a) is a truncation error term that enforces the charge-conserving flux be equal to the energy conserving flux, $\xi_{\alpha_{i+1 / 2}}^{k+1}$ will enforce the nonlinear constraint, and $\widehat{f}_{\alpha_{i+1 / 2, j}}^{k+1, *}$ is the numerical flux at the cell face. As before, the * denotes that the choice of numerical flux estimation can be different from the physical streaming operator. In order to determine the expression for $\xi_{\alpha_{i+1 / 2}}^{k+1}$, we introduce the following nonlinear constraint,

$$
\underbrace{\widehat{n u}_{\alpha_{i+1 / 2}}^{k+1 / 2}+\Xi_{\alpha_{i+1 / 2}}^{k+1}}_{\text {charge conserving flux }}=\underbrace{\widetilde{n u}_{\alpha_{i+1 / 2}}^{k+1 / 2}}_{\text {energy conserving flux }}
$$

where,

$$
\widehat{n u}_{\alpha_{i+1 / 2}}^{k+1 / 2}=\sum_{j=1}^{N_{v}} \Delta v_{\alpha_{j}} v_{\alpha_{j}} \widehat{f}_{\alpha_{i+1 / 2, j}}^{k+1 / 2}
$$




$$
\widetilde{n u}_{\alpha_{i+1 / 2}}^{k+1 / 2}=\frac{\sum_{j}^{N_{v}} \Delta v_{\alpha_{j+1 / 2}} v_{\alpha_{j+1 / 2}}\left(\Delta x_{i} \widetilde{f}_{\alpha_{i, j+1 / 2}}^{k+1 / 2}+\Delta x_{i+1} \widetilde{f}_{\alpha_{i+1, j+1 / 2}}^{k+1 / 2}\right)}{2 \Delta x_{i+1 / 2}}
$$

and

$$
\Xi_{\alpha_{i+1 / 2}}^{k+1}=\xi_{\alpha_{i+1 / 2}}^{k+1} \sum_{j}^{N_{v}} \Delta v_{\alpha_{j}}\left|v_{\alpha_{j}}\right| \widehat{f}_{\alpha_{i+1 / 2, j}}^{k+1, *}
$$

From equations (3.17), (3.18), and (3.19), we can solve for $\xi_{\alpha_{i+1 / 2}}^{k+1}$,

$$
\xi_{\alpha_{i+1 / 2}}^{k+1}=\frac{\widetilde{n u}_{\alpha_{i+1 / 2}}^{k+1 / 2}-\widehat{n u}_{\alpha_{i+1 / 2}}^{k+1 / 2}}{\sum_{j}^{N_{v}} \Delta v_{\alpha_{j}}\left|v_{\alpha_{j}}\right| \widehat{f}_{\alpha_{i+1 / 2, j}}^{k+1, *}} .
$$

Similarly to $\phi_{\alpha_{i}}^{k+1}, \xi_{\alpha_{i+1 / 2}}^{k+1}$ is inherently nonlinear, and requires an iterative scheme to converge the system, which we absorb in the HOLO iteration. We note that $\Xi_{\alpha_{i+1 / 2}}^{k+1}$ is the truncation error difference between $\widehat{n u}_{\alpha_{i+1 / 2}}^{k+1 / 2}$ and $\widetilde{n u}_{\alpha_{i+1 / 2}}^{k+1 / 2}$. In the continuum, $\Xi_{\alpha_{i+1 / 2}}^{k+1}=0$. The choice of $|v|$ (instead of $v$ ) in (a) ensures regularity of $\xi_{\alpha_{i+1 / 2}}^{k+1}$ by forcing the denominator in equation (3.20) to be positive definite.

\subsection{Charge, Momentum, and Energy Conserving Discretization}

We extend the nonlinear enslavement concept introduced earlier to enforce discrete charge, momentum, and energy conservation simultaneously. As introduced before, the modified discrete Vlasov-Ampère system reads:

$$
\begin{gathered}
\frac{f_{\alpha_{i, j}}^{k+1}-f_{\alpha_{i, j}}^{k}}{\Delta t}+v_{\alpha_{j}} \frac{\widehat{f}_{\alpha_{i+1 / 2, j}}^{k+1 / 2}-\widehat{f}_{\alpha_{i-1 / 2 j}}^{k+1}+\left|v_{\alpha_{j}}\right| \frac{\xi_{\alpha_{i+1 / 2}}^{k+1} \widehat{f}_{\alpha_{i+1 / 2, j}}^{k+1, *}-\xi_{\alpha_{i-1 / 2}}^{k+1} \widehat{f}_{\alpha_{i-1 / 2, j}}^{k+1, *}}{\Delta x_{i}}+}{\frac{q_{\alpha}}{m_{\alpha}} E_{i}^{k+1 / 2}\left(\frac{\widetilde{f}_{\alpha_{i, j+1 / 2}}^{k+1 / 2}-\widetilde{f}_{\alpha_{i, j}-1 / 2}^{k+1 / 2}}{\Delta v_{\alpha_{j}}}+\phi_{\alpha_{i}}^{k+1} \frac{\widetilde{f}_{\alpha_{i, j}+1 / 2}^{k+1, *}-\widetilde{f}_{\alpha_{i, j}-1 / 2}^{k+1, *}}{\Delta v_{\alpha_{j}}}\right)=0 .} \\
\epsilon_{0} \frac{E_{i+1 / 2}^{k+1}-E_{i+1 / 2}^{k}}{\Delta t}+\sum_{\alpha}^{N_{s}} q_{\alpha} n u_{E C, \alpha_{i+1 / 2}}^{k+1 / 2}=0 .
\end{gathered}
$$

The combined charge, momentum, and energy conservation requires a modification to the nonlinear constraints discussed in sections 3.1 and 3.2. The energy conserving flux is now defined as,

$n u_{E C, \alpha_{i+1 / 2}}^{k+1 / 2}=\frac{\sum_{j}^{N_{v}} \Delta v_{\alpha_{j+1 / 2}} v_{\alpha_{j+1 / 2}}\left[\Delta x_{i}\left(\widetilde{f}_{\alpha_{i, j+1 / 2}}^{k+1 / 2}+\phi_{\alpha_{i}}^{k+1} \widetilde{f}_{\alpha_{i, j+1 / 2}}^{k+1, *}\right)+\Delta x_{i+1}\left(\widetilde{f}_{\alpha_{i+1, j+1 / 2}}^{k+1 / 2}+\phi_{\alpha_{i+1}}^{k+1} \widetilde{f}_{\alpha_{i, j+1 / 2}}^{k+1, *}\right)\right]}{2 \Delta x_{i+1 / 2}}$,

with $\phi_{\alpha_{i}}^{k+1}$ defined by equation (3.13) and the charge conserving flux unchanged,

$$
\begin{array}{r}
n u_{C C, \alpha_{i+1 / 2}}^{k+1 / 2}=\widehat{n u}_{\alpha_{i+1 / 2}}^{k+1 / 2}+\Xi_{\alpha_{i+1 / 2}}^{k+1}= \\
\sum_{j}^{N_{v}} \Delta v_{\alpha_{j}} v_{\alpha_{j}} \widehat{f}_{\alpha_{i+1 / 2, j}}^{k+1 / 2}+\xi_{\alpha_{i+1 / 2}}^{k+1} \sum_{j}^{N_{v}} \Delta v_{\alpha_{j}}\left|v_{\alpha_{j}}\right| \widehat{f}_{\alpha_{i+1 / 2, j}}^{k+1, *} .
\end{array}
$$

Simultaneous conservation of charge, momentum, and energy requires:

$$
n u_{C C, \alpha_{i+1 / 2}}^{k+1 / 2}=n u_{E C, \alpha_{i+1 / 2}}^{k+1 / 2},
$$


which gives the following relation for $\xi_{\alpha_{i+1 / 2}}^{k+1}$,

$$
\xi_{\alpha_{i+1 / 2}}^{k+1}=\frac{n u_{E C, \alpha_{i+1 / 2}}^{k+1 / 2}-\widehat{n u}_{\alpha_{i+1 / 2}}^{k+1 / 2}}{\sum_{j}^{N_{v}} \Delta v_{\alpha_{j}}\left|v_{\alpha_{j}}\right| \widehat{f}_{\alpha_{i+1 / 2, j}}^{k+1, *}} .
$$

Again, the nonlinearity in both $\xi_{\alpha_{i+1 / 2}}^{k+1}$ and $\phi_{\alpha_{i+1 / 2}}^{k+1}$ will be absorbed in the HOLO iteration and the order of accuracy of the method is determined by the discretization of the physical operators.

In this study, for robustness, we adopt the QUICK discretization [17] for the spatial streaming flux $\widehat{f}_{\alpha_{i+1 / 2, j}}^{k+1 / 2}$,

$$
\widehat{f}_{\alpha_{i+1 / 2, j}}^{k+1 / 2}=\left\{\begin{array}{cc}
\frac{6}{8} f_{\alpha_{i, j}}^{k+1 / 2}+\frac{3}{8} f_{\alpha_{i+1, j}}^{k+1 / 2}-\frac{1}{8} f_{\alpha_{i-1, j}}^{k+1 / 2} & \text { if } v \geq 0 \\
\frac{6}{8} f_{\alpha_{i+1, j}}^{k+1 / 2}+\frac{3}{8} f_{\alpha_{i, j}}^{k+1 / 2}-\frac{1}{8} f_{\alpha_{i+2, j}}^{k+1 / 2} & \text { else }
\end{array}\right\} .
$$

The consistency operators only target the truncation error in the conservation property and do not affect the accuracy of the scheme. For robustness, an upwind discretization is used for the corresponding flux $\widehat{f}_{\alpha_{i+1 / 2, j}}^{k+1, *}$,

$$
\widehat{f}_{\alpha_{i+1 / 2, j}}^{k+1, *}=\left\{\begin{array}{cc}
f_{\alpha_{i, j}}^{k+1} & \text { if } v \geq 0 \\
f_{\alpha_{i+1, j}}^{k+1} & \text { else }
\end{array}\right\}
$$

Finally, central differencing is found to be sufficient for the velocity-space fluxes, $\widetilde{f}_{\alpha_{i, j+1 / 2}}^{k+1 / 2}$ and $\tilde{f}_{\alpha_{i, j+1 / 2}}^{k+1, *}$,

$$
\left(\widetilde{f}_{\alpha_{i, j+1 / 2}}^{k+1 / 2}, \widetilde{f}_{\alpha_{i, j}}^{*, k+1}\right)=\frac{1}{2}\left[\left(f_{\alpha, i, j}^{k+1 / 2}, f_{\alpha, i, j}^{k+1}\right)+\left(f_{\alpha, i+1, j}^{k+1 / 2}, f_{\alpha, i+1, j}^{k+1}\right)\right]
$$

The overall order accuracy of the proposed scheme is second-order. This will be shown numerically.

\section{Solver: HO and LO System}

We discuss the solver strategy for the coupled HOLO system. In this study, both the HO and LO system are solved fully implicitly using a Jacobian-Free Newton-Krylov (JFNK) method [16].

\subsection{HO System: Fully Implicit Charge, Momentum, and Energy Conserving ILU Preconditioned JFNK Solver}

The HO system is discretized with finite volumes on a collocated mesh, using a Crank-Nicolson timediscretization. The time, space, and velocity discretized, conservative Vlasov equation for species $\alpha$ reads:

$$
\begin{aligned}
\mathbb{R}_{v e, \alpha_{i, j}}=\frac{f_{\alpha_{i, j}}^{k+1, z}-f_{\alpha_{i, j}}^{k}}{\Delta t}+v_{\alpha_{j}} \frac{\widehat{f}_{\alpha_{i+1 / 2, j}}^{k+1 / 2, z}-\widehat{f}_{\alpha_{i-1 / 2, j}}^{k+1 / 2, z}}{\Delta x_{i}}+\left|v_{\alpha_{j}}\right| \frac{\xi_{\alpha_{i+1 / 2}}^{k+1, z-1} \widehat{f}_{\alpha_{i+1 / 2, j}}^{k+1, *, z}-\xi_{\alpha_{i-1 / 2}}^{k+1, z-1} \widehat{f}_{\alpha_{i-1 / 2, j}}^{k+1, *, z}}{\Delta x_{i}}+ \\
\frac{q_{\alpha}}{m_{\alpha}} E_{i}^{k+1 / 2, z}\left\{\frac{\widetilde{f}_{\alpha_{i, j+1 / 2}}^{k+1 / 2, z}-\widetilde{f}_{\alpha_{i, j-1 / 2}}^{k+1 / 2, z}}{\Delta v_{\alpha_{j}}}+\phi_{\alpha_{i}}^{k+1, z-1} \frac{\widetilde{f}_{\alpha_{i, j+1 / 2}}^{k+1, *, z}-\widetilde{f}_{\alpha_{i, j-1 / 2}}^{k+1, *, z}}{\Delta v_{\alpha_{j}}}\right\}=0 .
\end{aligned}
$$

ere, the additional superscript $z$ denotes the HOLO iteration index. We remind the reader that the electric field, $E$, comes from the solution to the LO system, and

$$
\phi_{\alpha_{i}}^{k+1, z}=\frac{n_{\alpha_{i}}^{H O, k+1 / 2, z}-\widetilde{n}_{\alpha_{i}}^{H O, k+1 / 2, z}}{\sum_{j=1}^{N_{v}} \Delta v_{j+1 / 2} \widetilde{f}_{\alpha_{i, j+1 / 2}}^{k+1, *, z}},
$$




$$
\begin{gathered}
\xi_{\alpha_{i+1 / 2}}^{k+1, z}=\frac{n u_{E C, \alpha_{i+1 / 2}}^{H O, k+1 / 2, z}-\widehat{n u}_{\alpha_{i+1 / 2}}^{H O, k+1 / 2, z}}{\sum_{j=1}^{N_{v}} \Delta v_{\alpha_{j}}\left|v_{\alpha_{j}}\right| \widehat{f}_{\alpha_{i+1 / 2, j}}^{k+1, *, z}}, \\
\widehat{n u}_{\alpha_{i+1 / 2}}^{H O, k+1 / 2, z}=\sum_{j}^{N_{v}} \Delta v_{\alpha_{j}} v_{\alpha_{j}} \widehat{f}_{\alpha_{i+1 / 2, j}}^{k+1 / 2},
\end{gathered}
$$

where the HO energy conserving flux is:

$n u_{E C, \alpha_{i+1 / 2}}^{H O, k+1 / 2, z}=\frac{\sum_{j=1}^{N_{v}} \Delta v_{\alpha_{j+1 / 2}} v_{\alpha_{j+1 / 2}}\left[\Delta x_{i}\left(\tilde{f}_{\alpha_{i, j+1 / 2}}^{k+1 / 2, z}+\phi_{\alpha_{i}}^{k+1, z-1} \tilde{f}_{\alpha_{i, j+1 / 2}}^{k+1, *, z-1}\right)+\Delta x_{i+1}\left(\tilde{f}_{\alpha_{i+1, j+1 / 2}}^{k+1 / 2, z}+\phi_{\alpha_{i+1}}^{k+1, z-1} \tilde{f}_{\alpha_{i, j+1 / 2}}^{k+1, *, z-1}\right)\right]}{2 \Delta x_{i+1 / 2}}$

the $\mathrm{HO}$ charge conserving density is:

$$
n_{\alpha_{i}}^{H O, k+1 / 2, z}=\sum_{j=1}^{N_{v}} \Delta v_{\alpha_{j}} f_{\alpha_{i, j}}^{k+1 / 2}
$$

and the $\mathrm{HO}$ momentum conserving density is:

$$
\widetilde{n}_{\alpha_{i}}^{H O, k+1 / 2, z}=\sum_{j=1}^{N_{v}} \Delta v_{\alpha_{j+1 / 2}} \widetilde{f}_{\alpha_{i, j+1 / 2}}^{k+1 / 2, z}
$$

Note that the evaluation of $\xi_{\alpha_{i+1 / 2}}^{k+1}$ and $\phi_{\alpha_{i+1 / 2}}^{k+1}$ is lagged to the previous HOLO iteration. For this study, the Vlasov equation for each species are solved for $f^{k+1, z}$ using JFNK with an ILU preconditioning with a relative convergence tolerance of $10^{-4}$ and an absolute convergence tolerance of $10^{-6}$.

\subsection{LO System: Physics Based Preconditioned JFNK Solver}

The LO system is discretized using staggered finite differences and a Crank-Nicolson time-discretization. The time and space discrete implicit LO system reads:

$$
\begin{gathered}
\mathbb{R}_{n_{\alpha_{i}}}=\frac{n_{\alpha_{i}}^{L O, k+1, z}-n_{\alpha_{i}}^{k}}{\Delta t}+\frac{n u_{E C, \alpha_{i+1 / 2}}^{L O, k+1 / 2, z}-n u_{E C, \alpha_{i-1 / 2}}^{L O, k+1 / 2, z}}{\Delta x_{i}}=0, \\
\mathbb{R}_{n u_{\alpha_{i+1 / 2}}}=\frac{n u_{E C, \alpha_{i+1 / 2}}^{L O, k+1, z}-n u_{E C, \alpha_{i+1 / 2}}^{L O, k}+\frac{n_{\alpha_{i+1}}^{L O, k+1 / 2, z} \widetilde{S}_{\alpha_{i+1}}^{H O, k+1 / 2, z}-n_{\alpha_{i}}^{L O, k+1 / 2, z} \widetilde{S}_{\alpha_{i}}^{H O, k+1 / 2, z}}{\Delta t}}{-\frac{q_{\alpha}}{m_{\alpha}} n_{\alpha_{i+1 / 2}}^{L O, k+1 / 2, z} E_{i+1 / 2}^{k+1 / 2, z}-\gamma_{n u_{\alpha_{i+1 / 2}}}^{H O, k+1, z}=0,} \\
\mathbb{R}_{E_{i+1 / 2}}=\epsilon_{0} \frac{E_{i+1 / 2}^{k+1}-E_{i+1 / 2}^{k}}{\Delta t}+\sum_{\alpha} q_{\alpha} n u_{E C, \alpha_{i+1 / 2}}^{L O, k+1, z}=0 .
\end{gathered}
$$

Here, the density normalized total stress-tensor, $\widetilde{S}^{H O, k+1 / 2, z}$, is computed from the HO solution,

$$
\widetilde{S}_{\alpha_{i}}^{H O, k+1 / 2, z}=\frac{\sum_{j=1}^{N_{v}} \Delta v_{\alpha_{j}} v_{\alpha_{j}}^{2} f_{\alpha_{i, j}}^{k+1 / 2, z}}{\sum_{j=1}^{N_{v}} \Delta v_{\alpha_{j}} f_{\alpha_{i, j}}^{k+1 / 2, z}},
$$


and the discrete consistency term, $\gamma_{n u_{\alpha_{i+1 / 2}}^{H O, k+1, z}}$, is computed as:

$$
\begin{aligned}
\gamma_{n u_{\alpha_{i+1 / 2}}}^{H O, k+1, z} & =\frac{n u_{E C, \alpha_{i+1 / 2}}^{H O, k+1, z}-n u_{E C, \alpha_{i+1 / 2}}^{H O, k}}{\Delta t}+\frac{n_{\alpha_{i+1}}^{H O, k+1 / 2, z} \widetilde{S}_{\alpha_{i+1}}^{H O, k+1 / 2, z}-n_{\alpha_{i}}^{H O, k+1 / 2, z} \widetilde{S}_{\alpha_{i}}^{H O, k+1 / 2, z}}{\Delta x_{i+1 / 2}} \\
& -\frac{q_{\alpha}}{m_{\alpha}} n_{\alpha_{i+1 / 2}}^{H O, k+1 / 2, z} E_{i+1 / 2}^{k+1 / 2, z},
\end{aligned}
$$

where $n_{\alpha_{i+1 / 2}}^{L O / H O, k+1 / 2, z}$ is computed by interpolating $n_{\alpha_{i}}^{L O / H O, k+1 / 2, z}$ from cell centers to cell faces. The LO system is solved fully nonlinearly using a physics-based-preconditioned JFNK solver for the complete residual vector,

$$
\overrightarrow{\mathbb{R}}=\left\{\begin{array}{c}
\mathbb{R}_{n_{e}} \\
\mathbb{R}_{n u_{e}} \\
\mathbb{R}_{n_{i}} \\
\mathbb{R}_{n u_{i}} \\
\mathbb{R}_{E}
\end{array}\right\}
$$

For details on the preconditioner, we refer the reader to references [15, 22]. For the Newton iteration, we use a relative convergence tolerance of $10^{-6}$ and an absolute tolerance of $10^{-7}$.

\subsection{HOLO Accelerator Algorithm with Charge-Momentum-and-Energy Con- serving Discretization}

To check the convergence of the Picard HOLO iteration, we measure the $L_{2}$-norm of the relative difference between the moments of the HO and LO solution,

$$
\text { reldiff } \mathcal{M}_{\mathcal{M}}^{z}=\left|\frac{\mathcal{M}^{H O, z}-\mathcal{M}^{L O, z}}{\mathcal{M}^{H O, z}}\right|_{2}
$$

where $\mathcal{M}$ is the specific moment of choice (i.e. $n$ or $n u$ ). We have chosen a convergence tolerance of $10^{-6}$ in this study.

We outline the algorithm for the fully implicit, charge, momentum, and energy conserving, moment accelerated Vlasov-Ampere solver in Algorithm 1.

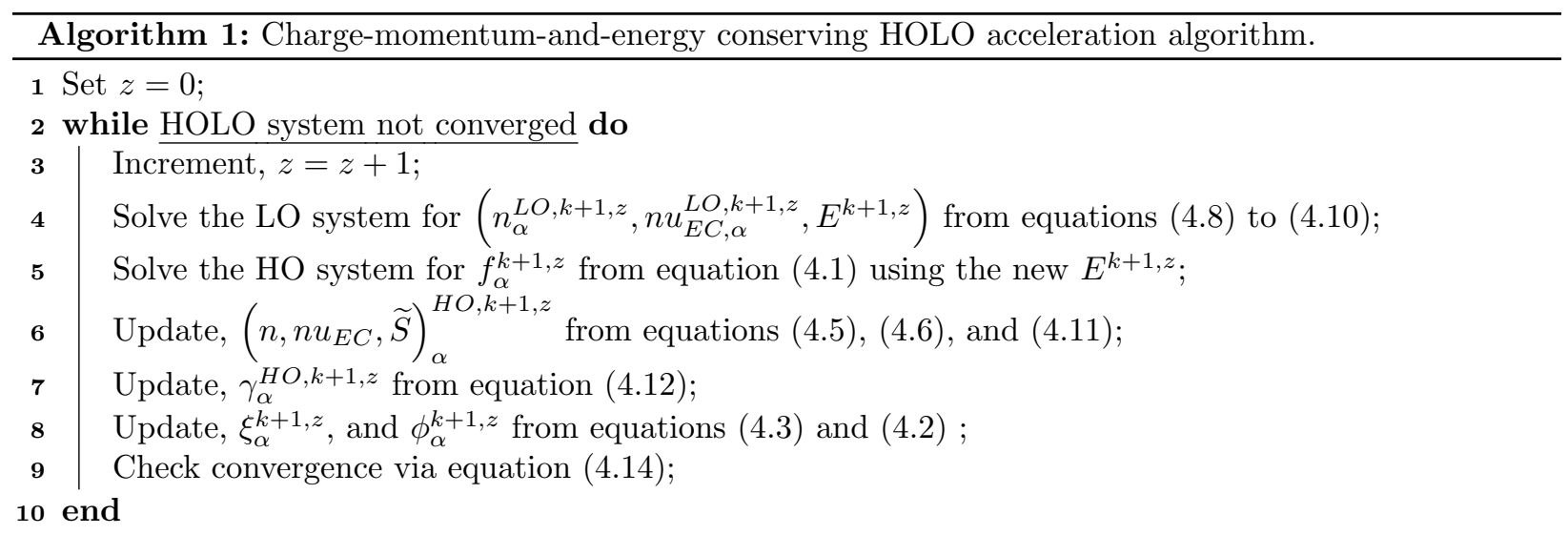

It is stressed here that the charge, momentum, and energy conserving discretization of the Vlasov equation and the HOLO iteration are two completely independent concepts. The conservation properties are built-in via the nonlinear discretization introduced, whereas the HOLO iteration is a nonlinear solution method. Thus, the HOLO algorithm is not an essential ingredient for to enforce conservation; any nonlinear solver that converges the residual will result in exact conservation. The advantage of coupling the conservative discretization of the Vlasov equation with the HOLO acceleration algorithm is that the nonlinearity in the discretization can be absorbed into the HOLO iteration and can be dealt with efficiently. 


\section{$5 \quad$ Numerical Results}

We present the algorithmic and accuracy performance results of the new HOLO solver with the proposed conservative discretization for the Vlasov-Ampere system. We choose the following test problems: 1) an electron Landau damping, 2) electron two-stream instability, and 3) an ion acoustic shockwave problem [21]. The purpose of these numerical tests is 1) to verify the correctness of the implementation, 2) to demonstrate the algorithmic ability to step over stiff time-scales and 3) to demonstrate the advertised conservation properties of the scheme in a periodic system.

For all of our test cases, unless otherwise specified, the distribution function is initialized according to the following initial condition:

$$
\begin{aligned}
f_{0}(x, v, u, T) & =\frac{n_{0}}{\sqrt{2 \pi T_{0} / m}} \exp \left[-\frac{m\left(v-u_{0}\right)^{2}}{2 T_{0}}\right], \\
n_{0} & =n_{0}(t=0, x)=1+\alpha_{n} \cos (k x),
\end{aligned}
$$

with the initial electric field found from Poisson's equation:

$$
\begin{gathered}
-\epsilon_{0} \frac{\partial^{2} \phi_{0}}{\partial x^{2}}=\sum_{\alpha}^{N_{s}} q_{\alpha} n_{\alpha_{0}}, \\
E_{0}=-\frac{\partial \phi_{0}}{\partial x} .
\end{gathered}
$$

Here $x, v, n_{0}, u_{0}, T_{0}, \alpha_{n}, k, m, \phi_{0}$, and $E_{0}$ are the position and velocity, initial number density, initial fluid velocity, initial temperature, perturbation amplitude for number density, wave vector of perturbation, the species mass, the initial electrostatic potential, and initial electric field, respectively.

\subsection{Landau Damping}

For the first test case, the classic single-species linear electron Landau damping problem is chosen. The rate at which the field energy is converted to the wave energy is found from the linear dispersion relation,

$$
1+\frac{1}{k^{2}}\left[1+\frac{\omega}{\sqrt{2} k} Z\left(\frac{\omega}{\sqrt{2} k}\right)\right]=0 .
$$

Here $k, \omega$, and $Z$ are the wave vector of perturbation, wave frequency and dispersion function of Fried and Conte, respectively. Solving for $\omega$, a complex solution is obtained in terms of the oscillatory (imaginary) $\tilde{\omega}$ and decaying (real) $\gamma$ components of the frequency. The problem parameters used for this single species test case are [19]: the periodic system length, $L_{x}=4 \pi$, the number of configuration cells, $N=32$, the velocity space domain, $v \in\left[-6 \sqrt{2},+6 \sqrt{2}\right.$, the number of velocity space cells, $N_{v}=512$, the maximum duration of simulation in terms of the electron plasma wave time-scale, $t_{\max }=600 \omega_{p, e}^{-1}$, the time-step size, $\Delta t=0.1 \omega_{p, e}^{-1}$, the wave vector of perturbation, $k=2 \pi / L_{x}$, the average density $n_{0}=1.0$, perturbation in density, $\alpha_{n}=0.01, u_{0}=0$, and temperature, $T_{0}=1.0$. For these given parameters, the growth rate is $\gamma$ $=-0.155$ [19]. The simulation result for the linear Landau damping case is shown in Figure 1. As can be seen, good agreement between the numerical simulation and linear theory is achieved until recurrence appears due to finite grid resolution effects. Several techniques have been proposed to suppress the recurrence effect $[10,11]$, although they were not considered in this study.

The new conservative discretization scheme adds a pseudo streaming and acceleration term in the discrete Vlasov equation. These terms are of the same order as the truncation error, and therefore they do not pollute the accuracy of the physical terms. To demonstrate this, a time, space, and velocity convergence study is performed. In Figure $2, L_{2}^{E, \Delta t}, L^{E, \Delta x}$, and $L^{E, \Delta v}$ are defined as:

$$
L_{2}^{E, \Delta}=\left|\left(\sum_{i}^{N_{x}} \Delta x_{i} E_{i}^{2}\right)-\left(\sum_{i}^{N_{x}} \Delta x_{i}\left(E_{i}^{\Delta, r e f}\right)^{2}\right)\right| .
$$



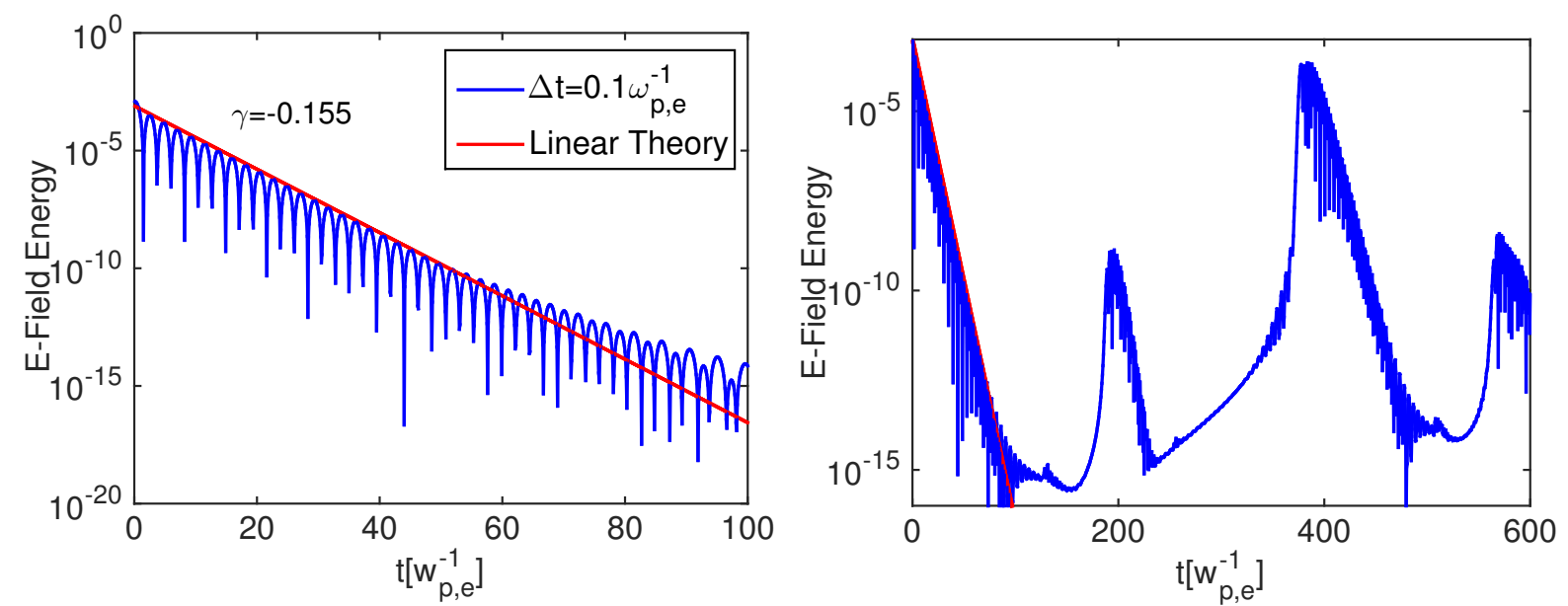

Figure 1: Decay rate comparison for linear Landau damping early on with $\Delta t=0.1 \omega_{p, e}^{-1}(\mathrm{left})$ and at longer time (right).
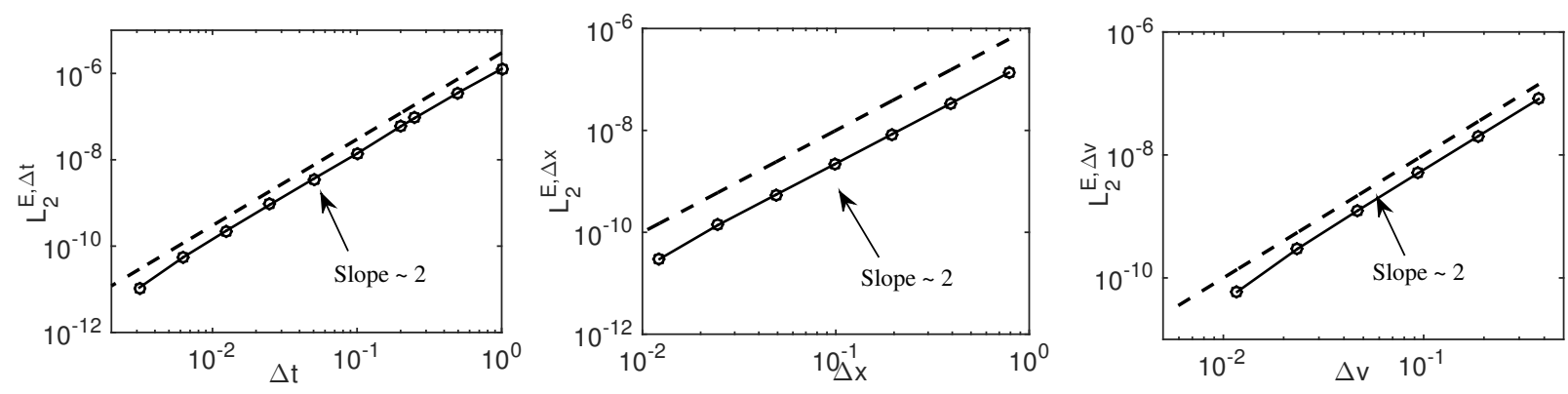

Figure 2: Time (left), space (center), and velocity (right) convergence study of the proposed scheme. The reference solution for the time step size convergence test was found with $t_{\max }=$ $1 \omega_{p, e}^{-1}, \Delta t^{r e f}=1.5626 \times 10^{-3} \omega_{p, e}^{-1}, N_{x}=32, N_{v}=512$. The reference solution for the configuration space cell size convergence test was found with $t_{\max }=1 \omega_{p, e}^{-1}, \Delta t=0.1 \omega_{p, e}^{-1}, N_{x}^{r e f}=2048, N_{v}=512$. The reference solution for the velocity space cell size convergence test was found with $t_{\max }=$ $1 \omega_{p, e}^{-1}, \Delta t=0.1 \omega_{p, e}^{-1}, N_{x}=32, N_{v}^{r e f}=2048$.

Here, the superscript $\Delta$, ref denotes reference quantities obtained from a simulation with a small time-step size, a high resolution mesh in configuration space, and a high resolution mesh in velocity space, respectively (see caption of Figure 2). As can be seen, a $2^{\text {nd }}$ order convergence is seen in time, space, and velocity as expected.

Finally, a strong, nonlinear Landau damping case was tested to study the accuracy of the solver with strong perturbations. We follow reference [6] and choose the following parameters: $\alpha=0.5, L_{x}=4 \pi, N_{x}=256, k_{x}=2 \pi / L_{x}, v \in[-6 \sqrt{2},+6 \sqrt{2}], N_{v}=512, \Delta t=0.1 \omega_{p, e}^{-1}, T_{0}=1$, and $t_{\max }=60\left[\omega_{p, e}^{-1}\right]$. In Figure 3, the electric field energy is shown as a function of time. As can be seen, the distinct damping and growth phases are captured with rates consistent with those reported in reference [6]. 


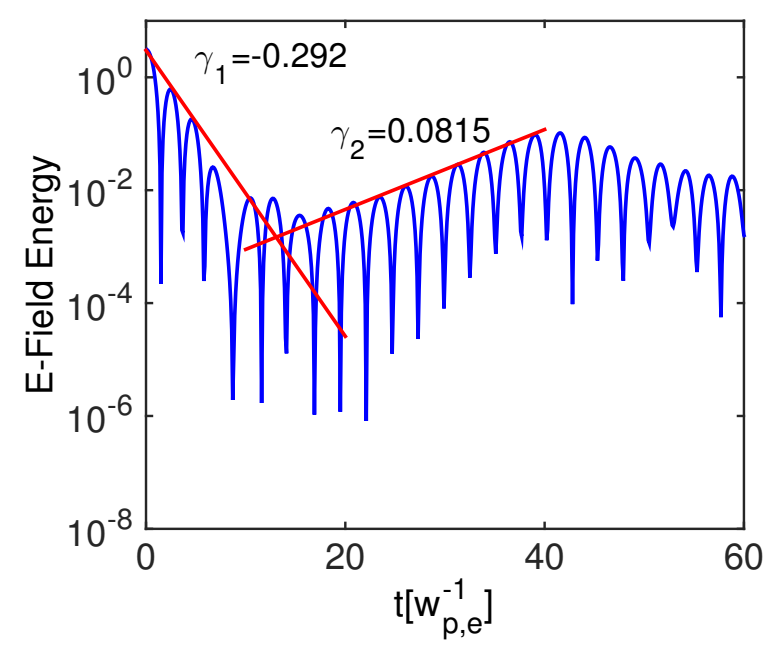

Figure 3: Decay and recurrence growth rate comparison for nonlinear Landau damping test case.

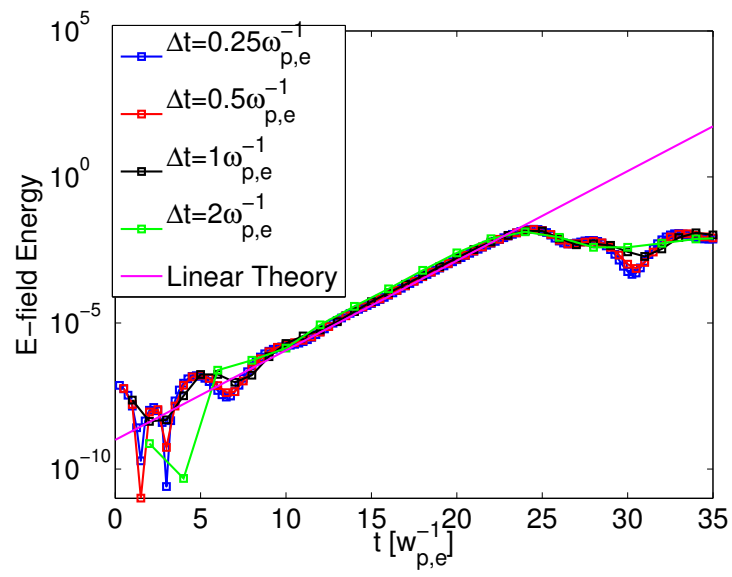

Figure 4: Electric field energy growth rate for two stream instability.

\subsection{Two-Stream Instability}

For the second benchmark case, we choose the single-species two-stream instability [20]. The dispersion relation for the two-stream instability is:

$$
1-\frac{1}{\left(\omega+v_{b} k\right)^{2}}-\frac{1}{\left(\omega-v_{b} k\right)^{2}}=0
$$

Here $v_{b}$ is the beam velocity and $\omega$ is again the wave frequency. The simulation is initialized as follows:

$$
f_{0}(x, v, t=0)=\left(1+\alpha_{n} \cos k x\right) \delta\left(v \pm v_{b}\right) .
$$

The simulation parameters are $L_{x}=1, N=32, t_{\max }=35 \omega_{p, e}^{-1}, \Delta t=0.25 \omega_{p, e}^{-1}, 0.5 \omega_{p, e}^{-1}, 1.0 \omega_{p, e}^{-1}, 2 \omega_{p, e}^{-1}$, $N_{v}=512, k=2 \pi / L_{x}, \alpha_{n}=0.001$, and $v_{b}=0.1$. From linear theory, the growth rate is $\gamma=0.353$. The comparison plot of the linear theory growth rate and numerical solutions for the electric field energy vs. time for various $\Delta t$ is shown in Figure 4. Again, good agreement between the linear theory and the numerical solution is observed in the linear growth regime. 


\begin{tabular}{|c|c|c|c|}
\hline$\Delta t / \omega_{p_{e}}^{-1}$ & 1 & 10 & 100 \\
\hline$\Delta t / \Delta t_{e x p}$ & $1.407 \times 10^{1}$ & $1.407 \times 10^{2}$ & $1.407 \times 10^{3}$ \\
\hline HOLO Iter $_{a v g}$ & 3.2 & 8.2 & 10.4 \\
\hline Picard Iter $_{a v g}$ & 7.8 & diverged & diverged \\
\hline
\end{tabular}

Table 2: HOLO solver performance with varying $\Delta t$ on a collisionless IASW problem.

\subsection{Ion Acoustic Shockwave (IASW) Problem}

For the IASW problem [21], the parameters are the electron mass, $m_{e}=1 / 1836$, ion mass of $m_{\text {ion }}=1$, Debye length, $\lambda_{D}=1 / 36$, system length, $L_{x}=144 \lambda_{D}$, number of configuration space cells, $N=144$, velocity space domain, $v_{\alpha_{\min }}=-10 v_{t h_{\alpha, 0}}, v_{\alpha_{\max }}=10 v_{t h_{\alpha, 0}}\left(v_{t h_{\alpha, 0}}=\sqrt{2 T_{\alpha_{0}} / m_{\alpha}}\right)$, number of velocity space cells, $N_{v}=400$, inverse ion plasma frequency, $\omega_{p, i o n}^{-1}=1 / 36$, inverse electron plasma frequency, $\omega_{p, e}^{-1}=\omega_{p, i o n}^{-1} / \sqrt{1836}=6.48 \times 10^{-4}$, and simulation duration, $t_{\max }=5000 w_{p, e}^{-1}$. For the initial condition, we use a sinusoidal perturbation in the ion density [21],

$$
n_{0, i o n}=1+0.2 \sin k x,
$$

electron density,

$$
n_{0, e}=1+0.2\left(1-k^{2} \lambda_{D}^{2}\right) \sin k x
$$

and bulk fluid velocity, $u$, for both species of the form:

$$
u_{0}=-1+0.2 \sin k x
$$

with isothermal distributions with $T_{0, e}=1$ and $T_{0, i o n}=0.05$. Here, $k=2 \pi / L_{x}$ is the wave vector of perturbation.

To demonstrate the algorithmic performance of the solver, we run the test case for time-step sizes $\Delta t=1 \omega_{p_{e}}^{-1}, 10 \omega_{p_{e}}^{-1}$, and $100 \omega_{p_{e}}^{-1}$ using the new HOLO algorithm and a standard Picard iteration algorithm (Appendix A). In Table 2, we show the implicit time-step-size relative to $\omega_{p, e}^{-1}$ and the explicit CFL constraint,

$$
\Delta t_{\exp }=\frac{\Delta x}{v_{e, \max }}
$$

the average number of HOLO iterations for convergence over the entire simulation, and the average number of Picard iterations for convergence over the entire simulation. We see that, for the IASW problem, the number of HOLO iterations is kept controlled for $\Delta t$ large relative to both $\omega_{p, e}^{-1}$ and the explicit time-step size while the Picard iteration scheme is divergent for $\Delta t>\omega_{p, e}^{-1}$.

We demonstrate next the advertised conservation properties of the scheme. For the field equation, we use the Ampère's equation. For charge conservation, it is critical that the discrete equivalence with the Poisson's equation be satisfied. For this reason, to measure charge conservation, we compute the $L_{2}$-norm of the discrete Gauss-law from time-step to time-step,

$$
|G L|_{2}=\left|\lambda_{D}^{2}\left(\frac{E_{i+1 / 2}^{k+1}-E_{i-1 / 2}^{k+1}}{\Delta x_{i}}-\frac{E_{i+1 / 2}^{k}-E_{i-1 / 2}^{k}}{\Delta x_{i}}\right)-\sum_{\alpha} q_{\alpha}\left(n_{\alpha_{i}}^{k+1}-n_{\alpha_{i}}^{k}\right)\right|_{2} .
$$

For momentum and energy, we show the relative difference of total energy and momentum with respect to the initial condition (denoted by the superscript 0 ):

$$
\begin{gathered}
\operatorname{reldiff}_{T E}^{k}=\left|\frac{T E^{0}-T E^{k}}{T E^{0}}\right|, \\
\operatorname{reldiff} T_{T M}^{k}=\left|\frac{T M^{0}-T M^{k}}{T M^{0}}\right| .
\end{gathered}
$$



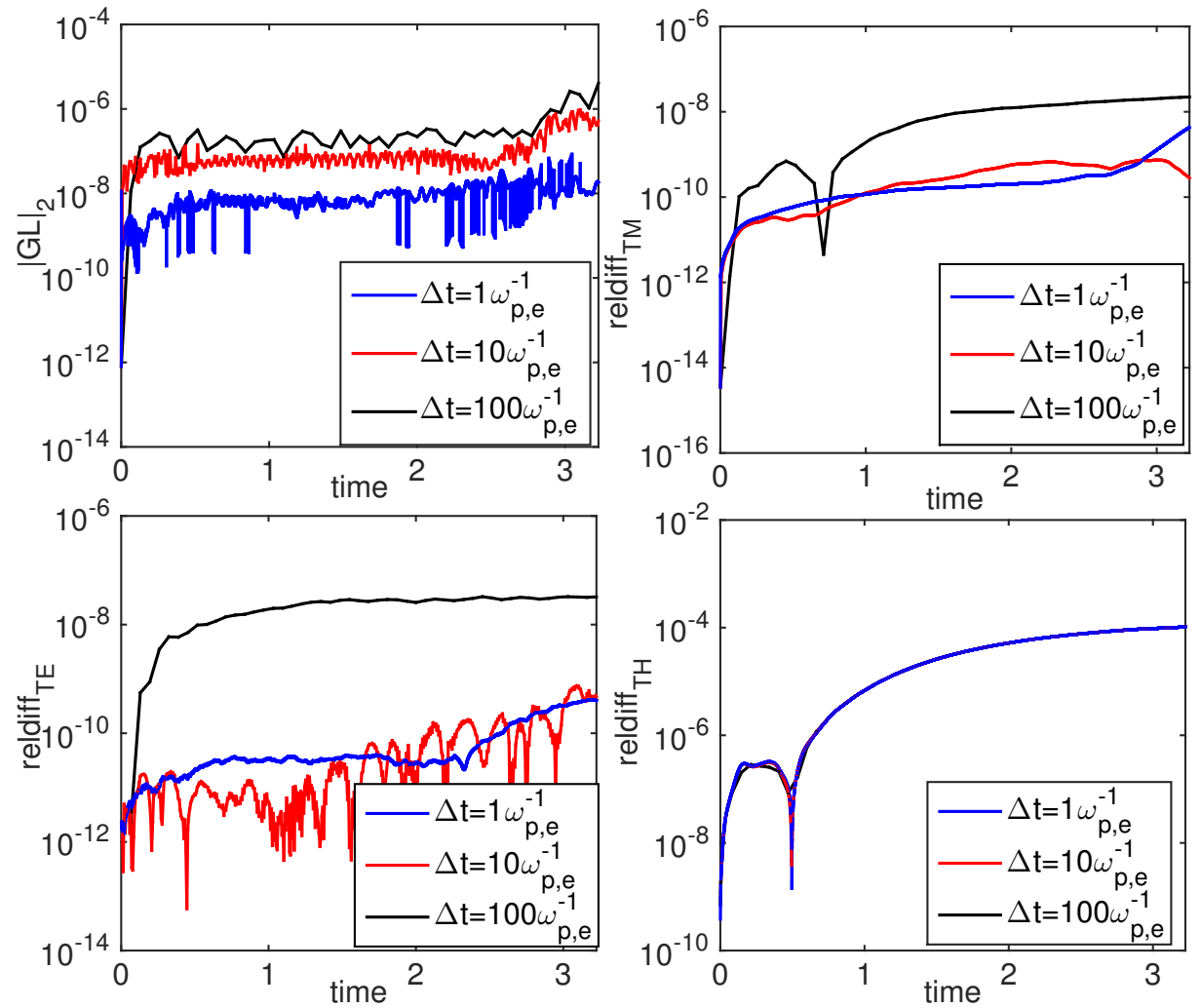

Figure 5: Conservation properties of the new charge-momentum-and-energy conserving discretization for various time step sizes.

For illustration purposes, we also show entropy conservation as a function of time (in the continuum, entropy is conserved by Vlasov equation),

$$
\text { reldiff } T_{T H}^{k}=\left|\frac{T H^{0}-T H^{k}}{T H^{0}}\right|
$$

where

$$
T H^{k}=-\sum_{\alpha}^{N_{s}} \sum_{i}^{N_{x}} \Delta x_{i} \sum_{j}^{N_{v}} \Delta v_{j} f_{\alpha_{i, j}}^{k} \ln \left|f_{\alpha_{i, j}}^{k}\right|,
$$

is the total entropy of the system. The conservation properties of the solver over the entire duration of the simulation for different time-step sizes are shown in Figure 5. We see that charge, momentum, and energy are conserved at or below the convergence tolerance of the HOLO iteration, tol $=10^{-6}$. The entropy conservation on the other hand is not enforced since the property is not built into the discretization. However, the error remains small $(\leq 0.01 \%)$ at all times. To establish accuracy, we compare the electron and ion densities, and electric field structure obtained for the different cases in Figure 6. As can be seen, the solutions with $\Delta t=10 \omega_{p, e}^{-1}$, and $100 \omega_{p, e}^{-1}$ are very similar to the $\Delta t=1 \omega_{p, e}^{-1}$ case.

\section{Conclusions}

In this study, we have 1) developed a charge, momentum, and energy conserving Vlasov-Ampère discretization, and 2) extended the HOLO moment-based acceleration algorithm for the Eulerian approach to solve 

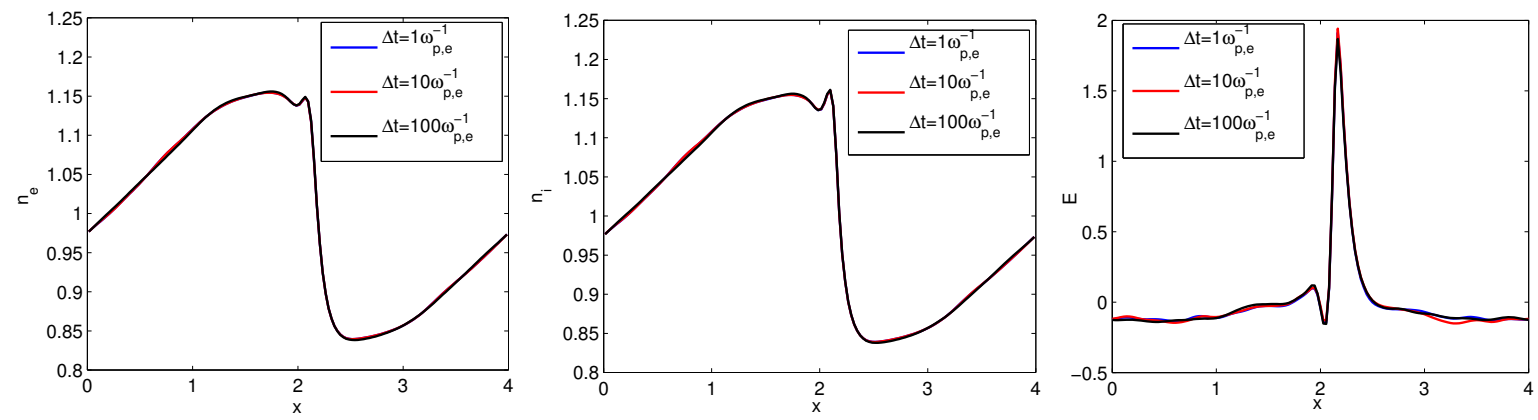

Figure 6: Solution for the ion acoustic shockwave at $t=5000 \omega_{p, e}^{-1}$ for various time step sizes

the Vlasov-Ampère system. In order to achieve the conservation properties, a novel nonlinear scheme is introduced which enslaves the momentum conserving density to the charge conserving density, and the charge conserving flux to the energy conserving flux. These enslavements were enforced by introducing two discrete nonlinear constraints, which resulted in a nonlinear discretization, which is dealt with efficiently by the HOLO iteration.

The algorithm has been tested on Landau damping, two-stream instability, and ion acoustic shockwave (IASW) problems. The Landau damping case demonstrated $2^{\text {nd }}$ order accuracy in time, configuration space, and velocity space. The IASW problem demonstrated that we can stably take a time-step-size, $\Delta t$, significantly larger than the inverse electron plasma frequency, $\omega_{p, e}^{-1}$, while evolving the solution accurately, with strong conservation properties of charge, momentum, and energy. Entropy is not exactly conserved by our scheme, but the error remains small and bounded.

\section{Acknowledgements}

The authors would like to acknowledge useful conversations with Dr. D. A. Knoll and Dr. G. Chen at the Los Alamos National Laboratory. This work was sponsored by the Los Alamos National Laboratory Directed Research and Development (LDRD) program. This work was performed under the auspices of the National Nuclear Security Administration of the U.S. Department of Energy at Los Alamos National Laboratory, managed by LANS, LLC under contract DE-AC52-06NA25396.

\section{References}

[1] J.W. Banks, J.A.F.Hittinger, "A new class of nonlinear finite-volume methods for Vlasov simulation", IEEE Trans. on Plasma Sci. 38, 9, (2010).

[2] T.D. Arber, R.C. VAnn, "A critical comparison of Eulerian-grid-based Vlasov solvers", J. Comput. Phys. 180 (2002) pp. 339-357.

[3] C.Z. Cheng, G. Knorr, "The integration of the Vlasov equation in configuration space", J. Comput. Phys. 22 (1976) pp. 330-351.

[4] R. Belaouar, N. Crouseilles, P. Degond, E. Sonnendrüker. "An Asymptotically Stable SemiLagrangian Scheme in the Quasi-Neutral Limit", J. Sci. Comput. 41 (2009) pp. 341-365.

[5] F. Filbet, E. Sonnendrücker, P. Bertrand, "Conservative numerical scheme for the Vlasov equation", J. Comput. Phys. 172 (2001) pp. 176-187.

[6] J.A. Rossmanith, D.C. Seal, "A positivity-preserving high-order semi-Lagrangian discontinuous Galerkin scheme for the Vlasov-Poisson equations", J. Comput. Phys. 230 (2011) pp. 6203-6232.

[7] R.B. Horne, M.P. Freeman, "A new code for electrostatic simulation by numerical integration of the Vlasov and Ampère's equations using MacCormack's method" J. Comput. Phys. 171 (2001), pp. 182-200. 
[8] Y. Cheng, A.J. Chrislieb, X.Zhong, "Energy-conserving discontinuous Galerkin methods for the Vlasov-Ampère system", J. Comput. Phys. 256 (2014) pp. 630-655.

[9] E.G. Evstatiev, B.A. Shadwick, "Variational formulation of particle algorithms for kinetic plasma simulations", J. Comput. Phys. 245 (2013) 376-398.

[10] H. Abbasi, M.H. Jenab, H. Hakimi Pajouh, "Preventing the recurrence effect in the Vlasov simulation by randomizing phase-point velocities in phase space", Phys. Rev. E 84 (2011) 036702.

[11] L. Einkemmer, A. Ostermann, "A strategy to suppress recurrence in gri-based Vlasov solvers", arXiv:1401.4809v1, 2014.

[12] W.T. Taitano, D.A. Knoll, L. Chacón, J. Reisner, A.K. Prinja, "Moment-based acceleration for neutral gas kinetics with BGK collision operator", TTSP accepted, 2014.

[13] D.A. Knoll, H. Park, K.S. Smith, "Application of the Jacobian-Free Newton-Krylov method to nonlinear acceleration of transport source iteration in slab geometry", Nuc. Sci. and Engn. 167 (2) (2011), pp. 122-132

[14] H. Park, D.A. Knoll, R.M. Rauenzahn, A.B. Wollaber, J.D. Densmore, "A Consistent, Moment-Based, Multiscale Solution Approach for Thermal Radiative Transfer Problems", Transport Theory and Statistical Physics, 41 (3-4) (2012), Special Issue: SI, pp. 284-303

[15] W.T. Taitano, D.A. Knoll, L. Chacón, G. Chen, "Development of a Consistent and Stable Fully Implicit Moment Method for Vlasov-Ampère Particle in Cell (PIC) System", SIAM J. Sci. Comput. 35-5 (2013), pp. 126-149, 2013.

[16] D.A. Knoll, D.E. Keyes, "Jacobian-Free Newton-Krylov Method: A survey of approaches and applications", J. Comput. Phys. 193 (2004) pp. 357-397.

[17] B.P. LEONARD, "A stable and accurate convective modeling procedure based on quadratic upstream interpolation", Comput. Meth. in Applied. Mech. and Eng. 19 (1979) pp. 59-98.

[18] B.P. LeOnard, S. Mokhtari, "Beyond First-Order Upwinding: The ULTRA-SHARP Alternative for Non-Oscillatory Steady-State Simulation of Convection", Int J. Num. Meth. Engng. 30 (1990) pp. 729-766.

[19] T.H. Watanabe, H. Suguma, "Vlasov and drift kinetic simulation methods based on the symplectic integrator", Trans. Theory and Stat. Phys. 34 (2005) pp. 287-309.

[20] F.F. Chen, "Introduction to Plasma Physics and Controlled Fusion, Volume 1: Plasma Physics", Springer Publishing (2006).

[21] M.A. Shay, J.F. Drake, B. Dorland, "Equation Free Projective Integration: A multiscale method applied to a plasma ion acoustic wave", J. Comput. Phys. 226 (2007) pp. 571-585.

[22] G. Chen, L. Chacón, C.A. Leibs, D.A. Knoll, W.Taitano, "Fluid preconditioning of NewtonKrylov-based, fully implicit, electrostatic particle-in-cell simulations", J. Comput. Phys. 258 (2014), pp. 555-567.

[23] G. Chen, L. Chacón, D.C. Barnes, "An energy- and charge-conserving, implicit, electrostatic particle-in-cell algorithm", J. Comput. Phys. 230 (2011), pp. 7018-7036.

[24] Y.W. Hou, Z.W. Ma, And M.Y. Yu "The plasma wave echo revisited", Phys. of Plas. 18, 012108, (2011).

[25] W.T. Taitano, D.A. Knoll, L. Chacón "Charge-and-Energy Conserving Moment-Based Accelerator for a Multi-Species Vlasov-Fokker-Planck-Ampère System, Part II: Collisional Aspects", J. Comput. Phys., in press, (2014). 


\section{A Appendix A: Picard Iteration Scheme for Solving a Coupled 1D1V Vlasov-Ampère System}

Consider the 1D1V Vlasov equation:

$$
\frac{\partial f_{\alpha}}{\partial t}+v \frac{\partial f_{\alpha}}{\partial x}+\frac{q_{\alpha}}{m_{\alpha}} \frac{\partial f_{\alpha}}{\partial v}=0
$$

and the 1D Ampère's equation:

$$
\epsilon_{0} \frac{\partial E}{\partial t}+\sum_{\alpha}^{N_{s}} q_{\alpha} n u_{\alpha}=0
$$

We solve the coupled system using a Picard iteration. For illustration purposes, we semi-discretize in time using a Crank-Nicholson scheme at time-step, $k$, and introduce a Picard iteration index, $z$, as:

$$
\frac{f_{\alpha}^{k+1, z}-f_{\alpha}^{k}}{\Delta t}+v \frac{\partial f_{\alpha}^{k+1 / 2, z}}{\partial x}+\frac{q_{\alpha}}{m_{\alpha}} E^{k+1 / 2, z-1} \frac{\partial f_{\alpha}^{k+1 / 2, z}}{\partial v}=0,
$$

and

$$
\frac{E^{k+1, z}-E^{k}}{\Delta t}+\sum_{\alpha}^{N_{s}} q_{\alpha} n u_{\alpha}^{k+1 / 2, z}=0,
$$

where

$$
n u_{\alpha}^{k+1 / 2, z}=\frac{1}{2}\left\langle v,\left(f_{\alpha}^{k+1, z}+f_{\alpha}^{k}\right)\right\rangle_{v}
$$

and

$$
E_{\alpha}^{k+1 / 2, z}=\frac{E^{k+1, z}+E^{k}}{2} .
$$

With a lagged evaluation for $E^{k+1 / 2, z-1}$, one can solve for $f_{\alpha}^{k+1, z}$ and evaluate a new $n u_{\alpha}^{k+1 / 2, z}$ to compute a new $E^{k+1 / 2, z}$. The iteration is continued until convergence, measured by the $L_{2}$-norm of the relative difference between the moments of consecutive iterations,

$$
\text { reldiff } \mathcal{M}_{\mathcal{M}}^{z}=\left|\frac{\mathcal{M}^{z}-\mathcal{M}^{z-1}}{\mathcal{M}^{z}}\right|_{2},
$$

where $\mathcal{M}$ is the specific moment of choice (i.e. $n$ or $n u$ ). We outline the algorithm for the Picard iteration scheme to solve for the Vlasov-Ampère system in Algorithm 2.

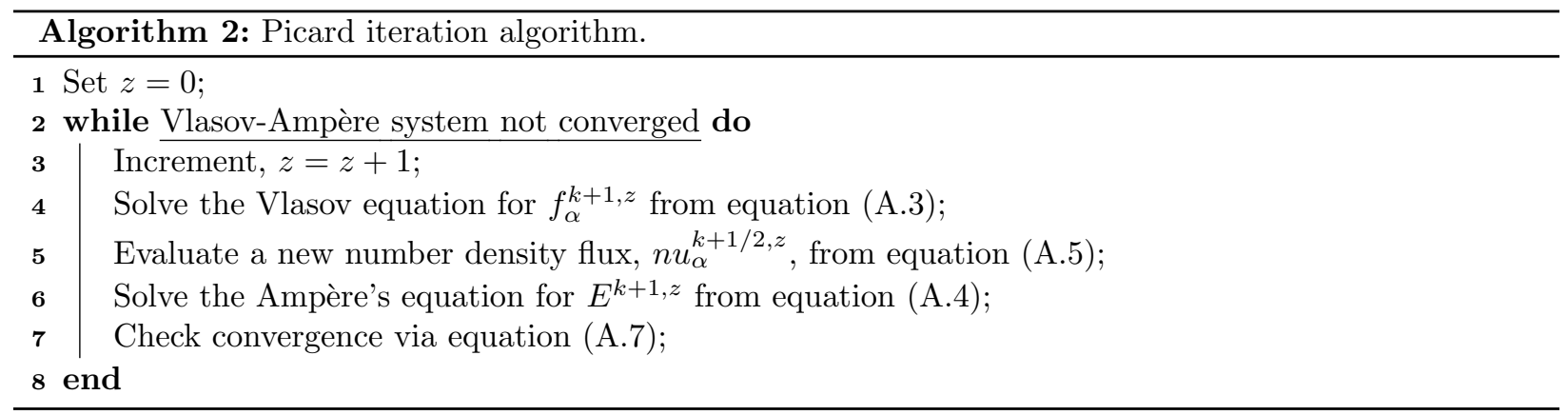

\section{B Appendix B: Independent Charge, Momentum, and Energy Conserving Discretization}

In the discrete, a particular definition for density, $n$, and number density flux, $n u$, is required to achieve separate conservations in charge, momentum, and energy. We derive the definitions of moments to achieve the separate conservation properties next. 


\section{B.1 Charge-Conserving Discretization}

Discrete charge conservation is enforced by satisfying the discrete equivalence between the Poisson's equation,

$$
\epsilon_{0} \frac{E_{i+1 / 2}^{k+1}-E_{i-1 / 2}^{k+1}}{\Delta x_{i}}-\sum_{\alpha}^{N_{s}} q_{\alpha} n_{\alpha_{i}}^{k+1}=0,
$$

and Ampère's equation,

$$
\epsilon_{0} \frac{E_{i+1 / 2}^{k+1}-E_{i+1 / 2}^{k}}{\Delta t}+\sum_{\alpha}^{N_{s}} q_{\alpha} n u_{\alpha_{i+1 / 2}}^{k+1 / 2}=0 .
$$

The conditions for discrete equivalence can be found by discretely differentiating equation (B.1) in time,

$$
\epsilon_{0} \frac{\left(E_{i+1 / 2}^{k+1}-E_{i+1 / 2}^{k}\right)-\left(E_{i-1 / 2}^{k+1}-E_{i-1 / 2}^{k}\right)}{\Delta t \Delta x_{i}}-\sum_{\alpha}^{N_{s}} q_{\alpha} \frac{n_{\alpha_{i}}^{k+1}-n_{\alpha_{i}}^{k}}{\Delta t}=0,
$$

discretely differentiating equation (B.2) in space,

$$
\epsilon_{0} \frac{\left(E_{i+1 / 2}^{k+1}-E_{i+1 / 2}^{k}\right)-\left(E_{i-1 / 2}^{k+1}-E_{i-1 / 2}^{k}\right)}{\Delta x_{i} \Delta t}+\sum_{\alpha}^{N_{s}} q_{\alpha} \frac{n u_{\alpha_{i+1 / 2}}^{k+1 / 2}-n u_{\alpha_{i-1 / 2}}^{k+1 / 2}}{\Delta x_{i}}=0
$$

and using the discrete $0^{\text {th }}$ moment of the Vlasov equation,

$$
\begin{aligned}
& \sum_{j}^{N_{v}} \Delta v_{j}\left\{\frac{f_{\alpha_{i, j}}^{k+1}-f_{\alpha_{i, j}}^{k}}{\Delta t}+v_{\alpha_{j}} \frac{\widehat{f}_{\alpha_{i+1 / 2, j}}^{k+1 / 2}-\widehat{f}_{\alpha_{i-1 / 2, j}}^{k+1 / 2}}{\Delta x_{i}}+\frac{q_{\alpha}}{m_{\alpha}} E_{i}^{k+1 / 2} \frac{\widetilde{f}_{\alpha_{i, j+1 / 2}}^{k+1 / 2}-\widetilde{f}_{\alpha_{i, j-1 / 2}}^{k+1 / 2}}{\Delta v_{\alpha_{j}}}\right\}= \\
& \frac{n_{\alpha_{i}}^{k+1}-n_{\alpha_{i}}^{k}}{\Delta t}+\frac{n u_{\alpha_{i+1 / 2}}^{k+1 / 2}-n u_{\alpha_{i-1 / 2}}^{k+1 / 2}}{\Delta x_{i}}=0 .
\end{aligned}
$$

From equations (B.3), (B.4), and (B.5), we see that discrete equivalence between the Poisson's equation and Ampère's equation (and thus charge conservation) is enforced by the following definitions for the number density,

$$
n_{\alpha_{i}}^{k+1}=\sum_{j}^{N_{v}} \Delta v_{\alpha_{j}} f_{\alpha_{i, j}}^{k+1}
$$

and number density flux,

$$
n u_{\alpha_{i+1 / 2}}^{k+1 / 2}=\sum_{j}^{N_{v}} \Delta v_{\alpha_{j}} v_{\alpha_{j}} \widehat{f}_{\alpha_{i+1 / 2, j}}^{k+1 / 2}
$$

\section{B.2 Momentum-Conserving Discretization}

Discrete momentum conservation is enforced by satisfying the discrete momentum balance of all species. In the discrete, the total momentum is defined by the sum of the species momentum,

$$
T M^{k+1}=\sum_{\alpha}^{N_{s}} m_{\alpha} \sum_{i}^{N} \Delta x_{i} n u_{\alpha_{i}}^{k+1},
$$

where $n u_{\alpha_{i}}^{k+1}$ is the cell-centered number density flux, defined as,

$$
n u_{\alpha_{i}}^{k+1}=\sum_{j}^{N_{v}} \Delta v_{\alpha_{j}} v_{\alpha_{j}} f_{\alpha_{i, j}}^{k+1} .
$$


The discrete momentum conservation is enforced when:

$$
T M^{k+1}-T M^{k}=\sum_{\alpha}^{N_{s}} m_{\alpha} \sum_{i}^{N} \Delta x_{i}\left(n u_{\alpha_{i}}^{k+1}-n u_{\alpha_{i}}^{k}\right)=0 .
$$

Substituting equation (B.9) into (B.10) and using equation (3.1), we obtain,

$$
\begin{array}{r}
T M^{k+1}-T M^{k}=\sum_{\alpha}^{N_{s}} m_{\alpha} \sum_{i=1}^{N} \Delta x_{i} \sum_{j}^{N_{v}} \Delta v_{\alpha_{j}} v_{\alpha_{j}}\left(f_{\alpha_{i, j}}^{k+1}-f_{\alpha_{i, j}}^{k}\right)= \\
-\Delta t \sum_{\alpha}^{N_{s}} m_{\alpha} \sum_{i=1}^{N} \Delta x_{i} \sum_{j}^{N_{v}} \Delta v_{\alpha_{j}} v_{\alpha_{j}}(\underbrace{v_{\alpha_{j}}^{\widehat{f}_{\alpha_{i+1 / 2, j}}^{k+1 / 2}-\widehat{f}_{\alpha_{i-1 / 2, j}}^{k+1 / 2}}}_{\text {(a) }}+\frac{q_{\alpha}}{m_{\alpha}} E_{i}^{k+1 / 2} \frac{\widetilde{f}_{\alpha_{i, j}+1 / 2}^{k+1 / 2}-\widetilde{f}_{\alpha_{i, j}-1 / 2}^{k+1 / 2}}{\Delta v_{\alpha_{j}}}) .
\end{array}
$$

With periodic boundary conditions, term (a) vanishes exactly when summed over all configuration space cells. The expression in equation (B.11) simplifies to,

$$
T M^{k+1}-T M^{k}=-\Delta t \sum_{\alpha}^{N_{s}} \sum_{i=1}^{N} \Delta x_{i} \sum_{j}^{N_{v}} v_{\alpha_{j}} q_{\alpha} E_{i}^{k+1 / 2}\left(\widetilde{f}_{\alpha_{i, j+1 / 2}}^{k+1 / 2}-\widetilde{f}_{\alpha_{i, j-1 / 2}}^{k+1 / 2}\right) .
$$

Now, telescoping the velocity domain sum and using the identity,

$$
v_{\alpha_{j}}-v_{\alpha_{j+1}}=-\Delta v_{\alpha_{j+1 / 2}}
$$

we obtain,

$$
T M^{k+1}-T M^{k}=\Delta t \sum_{\alpha}^{N_{s}} q_{\alpha} \sum_{i=1}^{N} \Delta x_{i} E_{i}^{k+1 / 2} n_{\alpha_{i}}^{k+1 / 2},
$$

where we have defined the density at time level $k+1 / 2, n_{\alpha_{i}}^{k+1 / 2}$, in equation (B.14) as:

$$
n_{\alpha_{i}}^{k+1 / 2}=\sum_{j}^{N_{v}} \Delta v_{\alpha_{j+1 / 2}} \widetilde{f}_{\alpha_{i, j+1 / 2}}^{k+1 / 2}
$$

Now, we use the discrete Gauss' law (Poisson's equation), at time level $k+1 / 2$ :

$$
\epsilon_{0} \frac{E_{i+1 / 2}^{k+1 / 2}-E_{i-1 / 2}^{k+1 / 2}}{\Delta x_{i}}=\sum_{\alpha}^{N_{s}} q_{\alpha} n_{\alpha_{i}}^{k+1 / 2}
$$

and substitute into equation (B.14), to find:

$$
T M^{k+1}-T M^{k}=-\Delta t \epsilon_{0} \sum_{\alpha}^{N_{s}} \sum_{i=1}^{N} E_{i}^{k+1 / 2}\left(E_{i+1 / 2}^{k+1 / 2}-E_{i-1 / 2}^{k+1 / 2}\right),
$$

which, with equation (3.3) and for a periodic system, finally gives:

$$
T M^{k+1}-T M^{k}=\Delta t \frac{\epsilon_{0}}{2} \sum_{i=1}^{N}\left[\left(E_{i+1 / 2}^{k+1 / 2}\right)^{2}-\left(E_{i-1 / 2}^{k+1 / 2}\right)^{2}\right]=0 .
$$

The critical step in the analysis is equation (B.16). 


\section{B.3 Energy-Conserving Discretization}

The total energy is defined by the sum of the kinetic energy and field energy. We begin by multiplying equation (3.1) by $m_{\alpha}, \Delta t$, and take the second velocity moment to obtain:

$$
\begin{array}{r}
\Delta t m_{\alpha} \sum_{i}^{N} \Delta x_{i} \sum_{j}^{N_{v}} \Delta v_{\alpha_{j}} \frac{v_{\alpha_{j}}^{2}}{2}\{\frac{f_{\alpha_{i, j}}^{k+1}-f_{\alpha_{i, j}}^{k}}{\Delta t}+\underbrace{\left.v_{\alpha_{j}} \frac{\widehat{f}_{\alpha_{i+1 / 2, j}}^{k+1 / 2}-\widehat{f}_{\alpha_{i-1 / 2, j}}^{k+1 / 2}}{\Delta x_{i}}+\frac{q_{\alpha}}{m_{\alpha}} E_{i}^{k+1 / 2} \frac{\widehat{f}_{\alpha_{i, j}+1 / 2}^{k+1 / 2}-\widehat{f}_{\alpha_{i, j-1 / 2}}^{k+1 / 2}}{\Delta v_{\alpha_{j}}}\right\}=}_{\text {(a) }} \\
\sum_{i}^{N} \Delta x_{i}\left\{m_{\alpha}\left(U_{\alpha_{i}}^{k+1}-U_{\alpha_{i}}^{k}\right)-\Delta t q_{\alpha} n u_{\alpha_{i}}^{k+1 / 2} E_{i}^{k+1 / 2}\right\}=0,
\end{array}
$$

where, (a) vanishes due to periodic boundary conditions. Here, $U_{\alpha_{i}}^{k+1}$ is the total energy density of species $\alpha$ at cell $i$,

$$
U_{\alpha_{i}}^{k+1}=\sum_{j}^{N_{v}} \Delta v_{\alpha_{j}} \frac{v_{\alpha_{j}}^{2}}{2} f_{\alpha_{i, j}}^{k+1}
$$

and $n u_{\alpha_{i}}^{k+1 / 2}$ is the number density flux at cell centers, defined as,

$$
n u_{\alpha_{i}}^{k+1 / 2}=-\sum_{j}^{N_{v}} \Delta \gamma_{\alpha_{j}} \frac{v_{\alpha_{j}}^{2}}{2} \frac{\tilde{f}_{\alpha_{i, j+1 / 2}}^{k+1 / 2}-\widetilde{f}_{\alpha_{i, j-1 / 2}}^{k+1 / 2}}{\Delta \psi_{\alpha_{j}}} .
$$

Telescoping the sum and using the following identities,

$$
\begin{gathered}
v_{\alpha_{j}}^{2}-v_{\alpha_{j+1}}^{2}=\left(v_{\alpha_{j}}-v_{\alpha_{j+1}}\right)\left(v_{\alpha_{j}}+v_{\alpha_{j+1}}\right), \\
v_{\alpha_{j}}-v_{\alpha_{j+1}}=-\Delta v_{j+1 / 2},
\end{gathered}
$$

we obtain:

$$
n u_{\alpha_{i}}^{k+1 / 2}=\sum_{j}^{N_{v}} \Delta v_{\alpha_{j+1 / 2}} v_{\alpha_{j+1 / 2}} \widetilde{f}_{\alpha_{i, j+1 / 2}}^{k+1 / 2} .
$$

We sum equation (B.19) over all species,

$$
\sum_{\alpha}^{N_{s}} \sum_{i}^{N} \Delta x_{i}\left\{m_{\alpha}\left(U_{\alpha_{i}}^{k+1}-U_{\alpha}^{k}\right)-\Delta t q_{\alpha} n u_{\alpha_{i}}^{k+1 / 2} E_{i}^{k+1 / 2}\right\}=0,
$$

which we rearrange to obtain:

$$
\sum_{\alpha}^{N_{s}} \sum_{i}^{N} \Delta x_{i} m_{\alpha}\left(U_{\alpha_{i}}^{k+1}-U_{\alpha_{i}}^{k}\right)=\sum_{\alpha}^{N_{s}} \sum_{i}^{N} \Delta x_{i} \Delta t q_{\alpha} n u_{\alpha_{i}}^{k+1 / 2} E_{i}^{k+1 / 2} .
$$

Since the field is defined at cell faces, we express $E_{i}^{k+1 / 2}$ in terms of cell-face quantities to obtain:

$$
\sum_{\alpha}^{N_{s}} \sum_{i}^{N} \Delta x_{i} m_{\alpha}\left(U_{\alpha_{i}}^{k+1}-U_{\alpha_{i}}^{k}\right)=\sum_{\alpha}^{N_{s}} \sum_{i}^{N} \Delta x_{i} \Delta t q_{\alpha} n u_{\alpha_{i}}^{k+1 / 2} \frac{E_{i+1 / 2}^{k+1 / 2}+E_{i-1 / 2}^{k+1 / 2}}{2} .
$$

Now, we telescope the sum on the RHS to obtain:

$$
\sum_{\alpha}^{N_{s}} \sum_{i}^{N} \Delta x_{i} m_{\alpha}\left(U_{\alpha_{i}}^{k+1}-U_{\alpha_{i}}^{k}\right)=-\sum_{\alpha}^{N_{s}} \sum_{i}^{N} \Delta x_{i+1 / 2} \Delta t q_{\alpha} n u_{\alpha_{i+1 / 2}}^{k+1 / 2} E_{i+1 / 2}^{k+1 / 2},
$$


where:

$$
n u_{\alpha_{i+1 / 2}}^{k+1 / 2}=\frac{\Delta x_{i} n u_{\alpha_{i}}^{k+1 / 2}+\Delta x_{i+1} n u_{\alpha_{i+1}}^{k+1 / 2}}{2 \Delta x_{i+1 / 2}},
$$

with $\Delta x_{i+1 / 2}=x_{i+1}-x_{i}$. Finally, using equation (B.2) and solving for $\Delta t \sum_{\alpha}^{N_{s}} q_{\alpha} n u_{\alpha_{i+1 / 2}}^{k+1 / 2}$,

$$
\Delta t \sum_{\alpha}^{N_{s}} q_{\alpha} n u_{\alpha_{i+1 / 2}}^{k+1 / 2}=-\epsilon_{0}\left(E_{i+1 / 2}^{k+1}-E_{i+1 / 2}^{k}\right),
$$

and substituting into equation (B.28), we can show with a few additional algebraic manipulations that,

$$
\sum_{\alpha}^{N_{s}} \sum_{i}^{N} \Delta x_{i} m_{\alpha}\left(U_{\alpha_{i}}^{k+1}-U_{\alpha_{i}}^{k}\right)=-\frac{\epsilon_{0}}{2} \sum_{i}^{N} \Delta x_{i+1 / 2}\left[\left(E_{i+1 / 2}^{k+1}\right)^{2}-\left(E_{i+1 / 2}^{k}\right)^{2}\right] .
$$

This is a statement of discrete total energy conservation between the plasma species and field. It follows that the total energy is defined as:

$$
T E^{k+1}=\sum_{\alpha}^{N_{s}} \sum_{i}^{N} \Delta x_{i} m_{\alpha} U_{\alpha_{i}}^{k+1}+\frac{\epsilon_{0}}{2} \sum_{i}^{N} \Delta x_{i+1 / 2}\left(E_{i+1 / 2}^{k+1}\right)^{2} .
$$

The critical steps in the analysis are equations B.24 and B.29. 

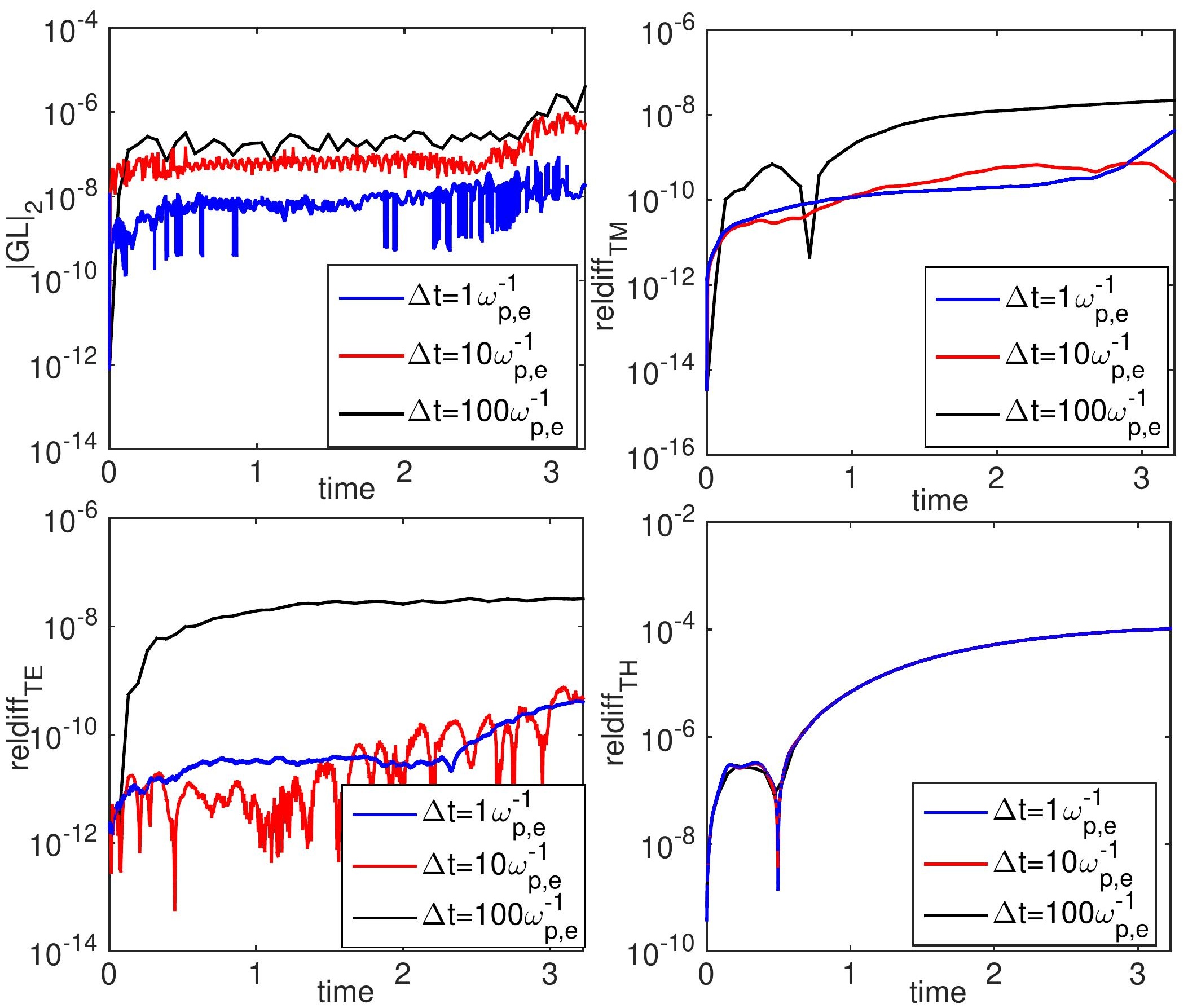


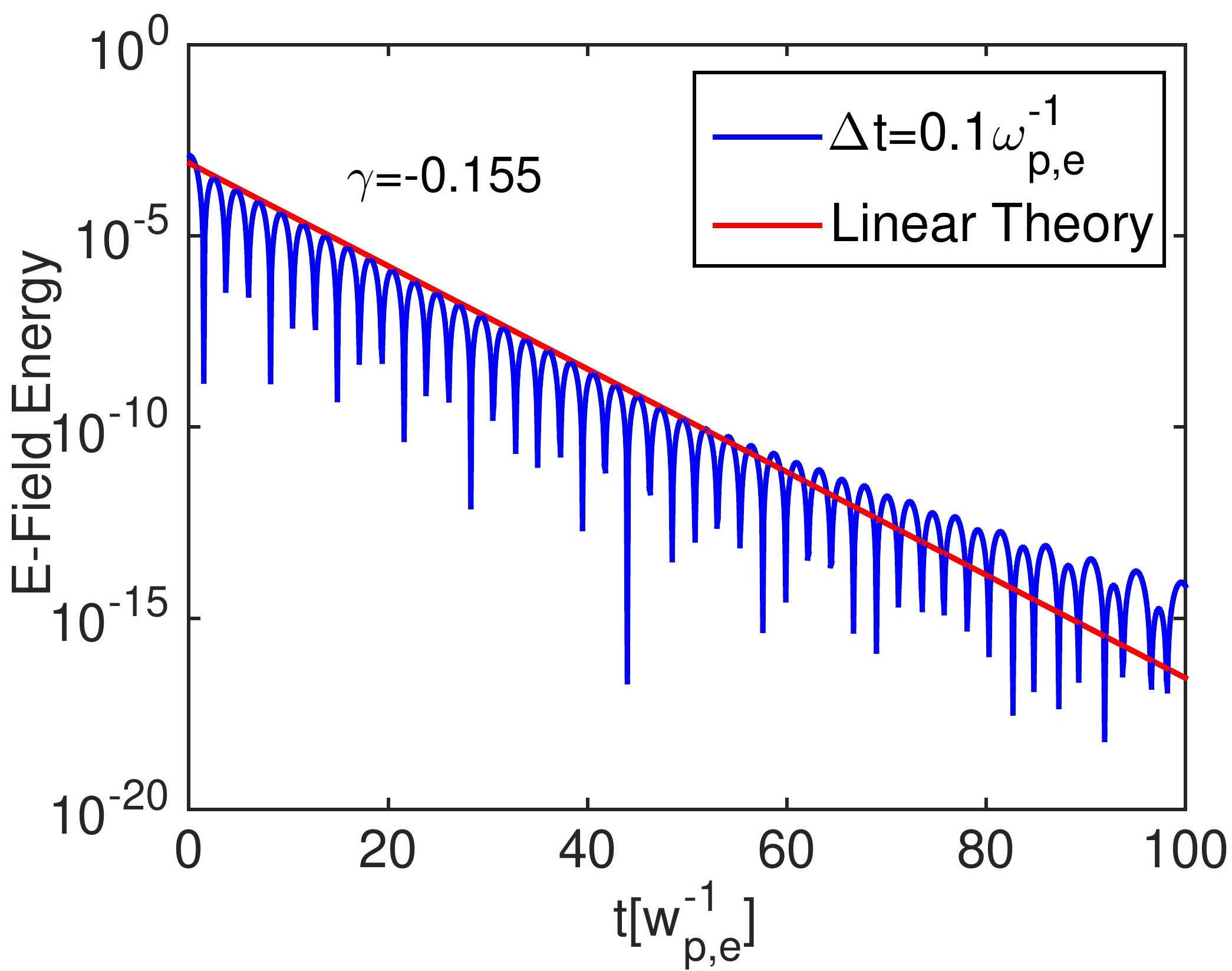




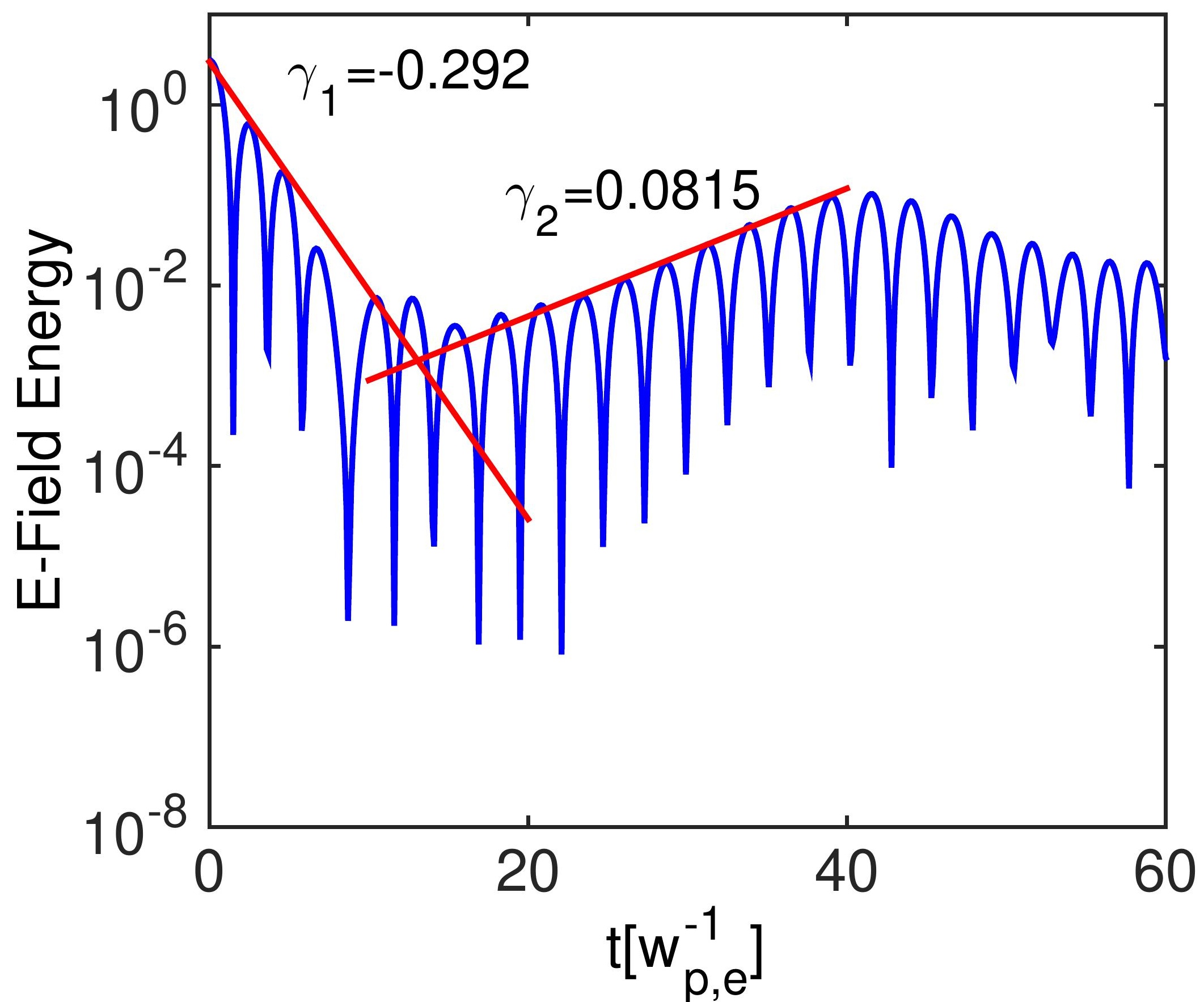




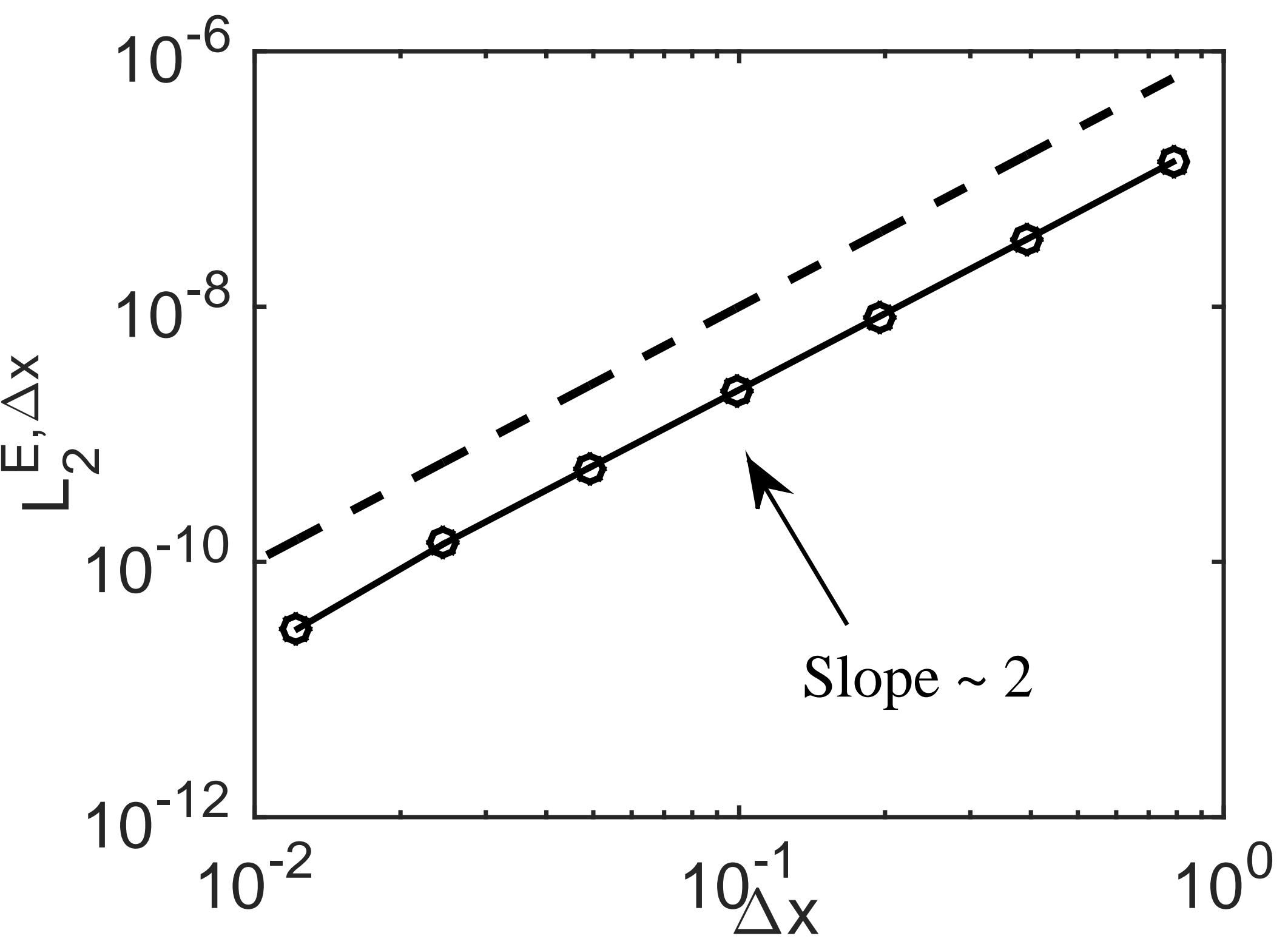




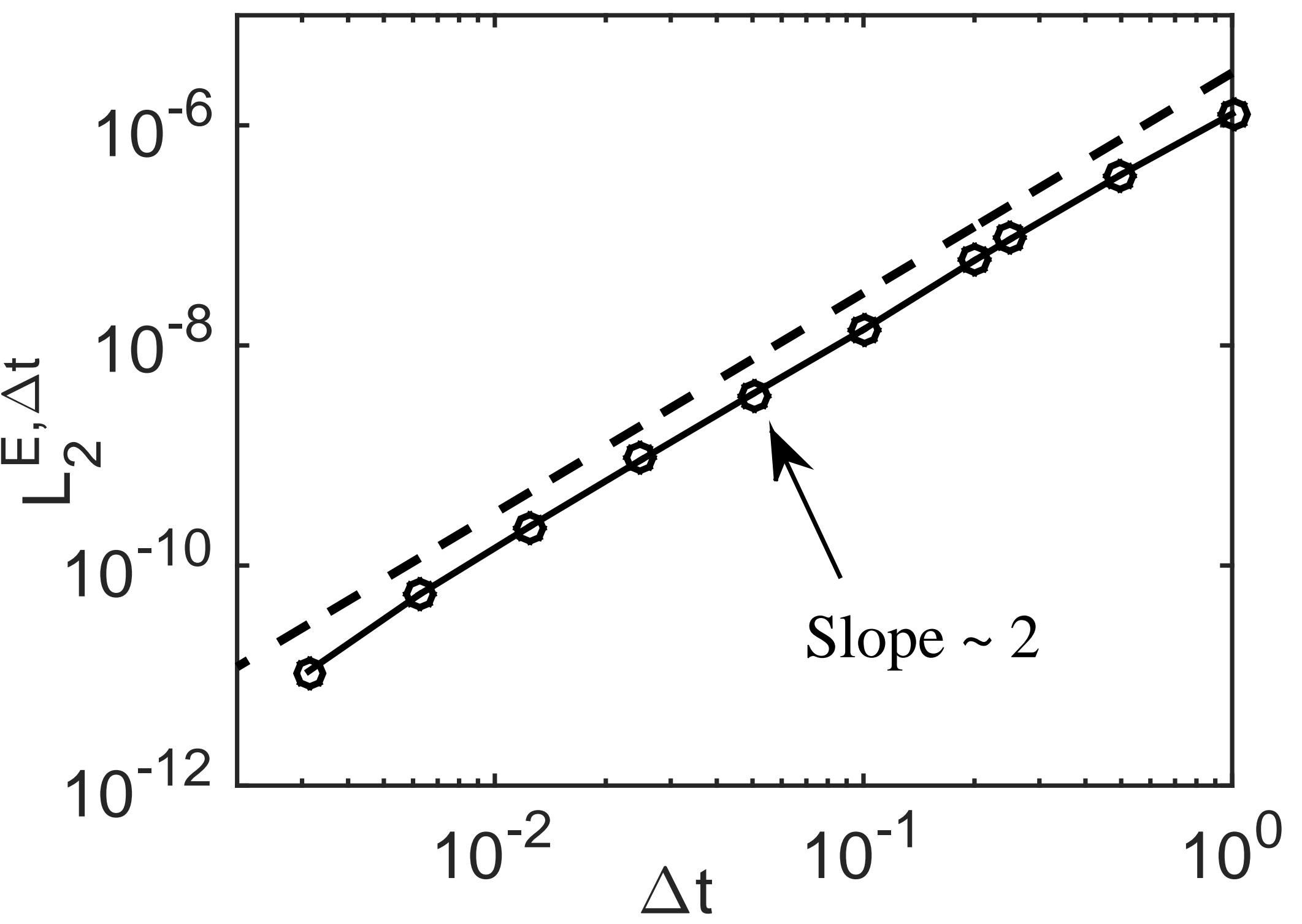




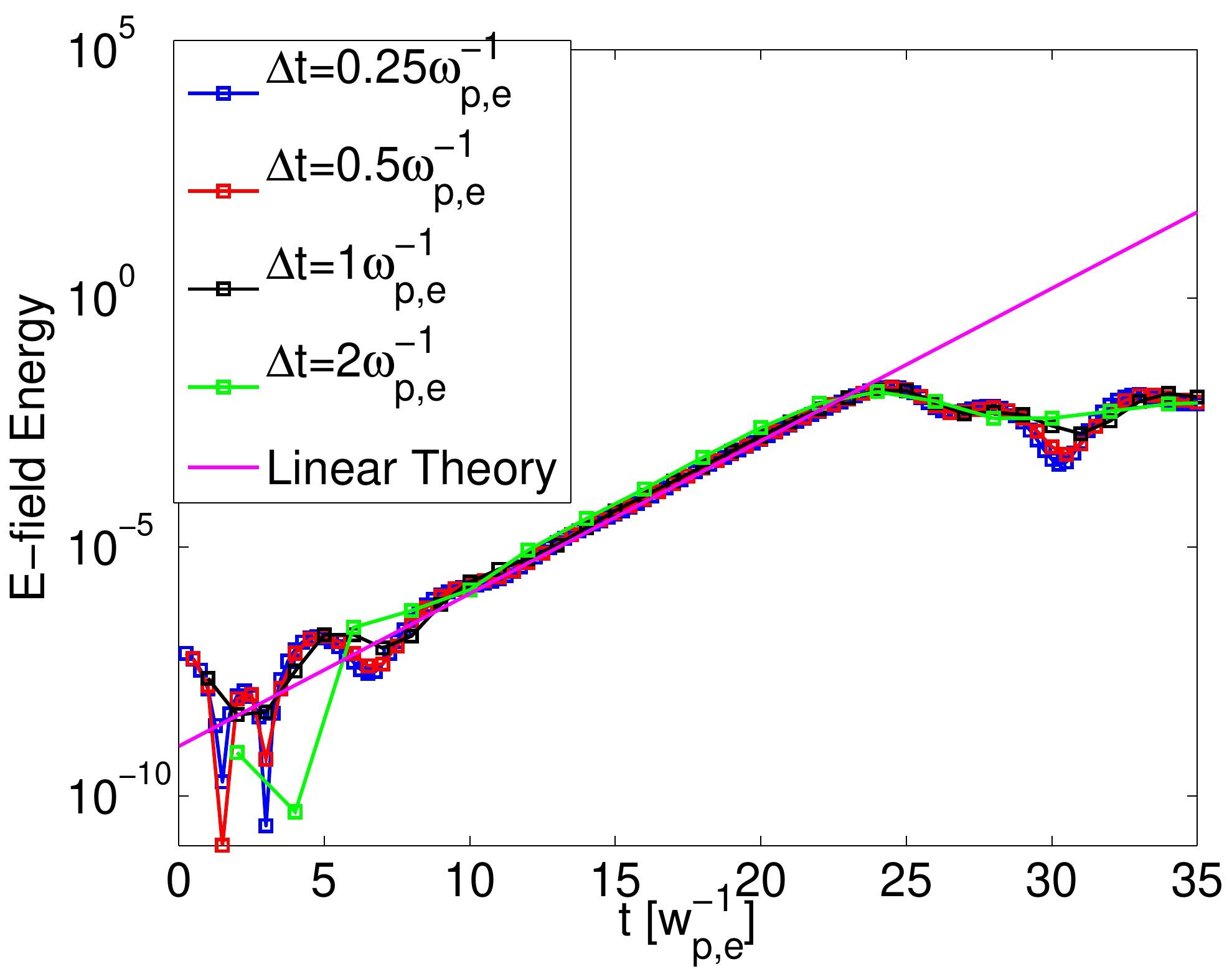




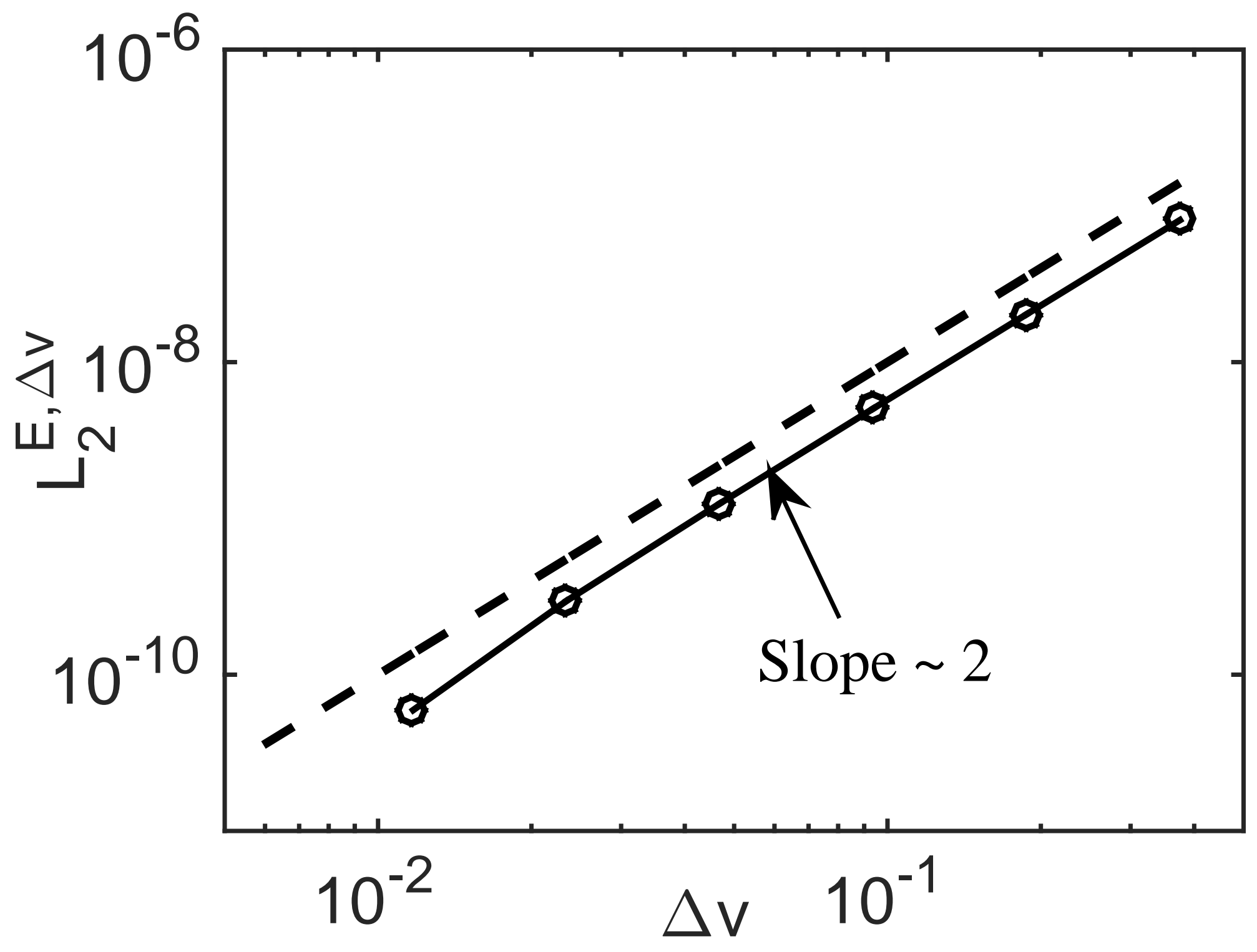

